\title{
Nano Based Approach for the Treatment of Neglected Tropical Diseases
}

\author{
Sureshbabu Ram Kumar Pandian ${ }^{1}$, Theivendren Panneerselvam ${ }^{2}$, Parasuraman Pavadai ${ }^{3}$, \\ Saravanan Govindaraj ${ }^{4}$, Vigneshwaran Ravishankar ${ }^{5}$, Ponnusamy Palanisamy ${ }^{6}$, \\ Muthukumar Sampath ${ }^{7}$, Murugesan Sankaranarayanan ${ }^{8 *}$ and Selvaraj Kunjiappan ${ }^{1 *}$
}

${ }^{1}$ Department of Biotechnology, Kalasalingam Academy of Research and Education, Krishnankoil, India, ${ }^{2}$ Department of Pharmaceutical Chemistry, Swamy Vivekanandha College of Pharmacy, Elayampalayam, India, ${ }^{3}$ Department of Pharmaceutical Chemistry, Faculty of Pharmacy, M. S. Ramaiah University of Applied Sciences, Bengaluru, India, ${ }^{4}$ Department of Pharmaceutical Chemistry, MNR College of Pharmacy, Sangareddy, India, ${ }^{5}$ Department of Biotechnology, Mepco Schlenk Engineering College, Sivakasi, India, ${ }^{6}$ School of Mechanical Engineering, Vellore Institute of Technology, Vellore, India, ${ }^{7}$ Department of Bioengineering, Birla Institute of Technology Mesra, Ranchi, India, ${ }^{8}$ Department of Pharmacy, Birla Institute of Technology and Science Pilani, Pilani, India

OPEN ACCESS

Edited by:

Kandasamy Saravanakumar, Kangwon National University, South

Korea

Reviewed by: Vijayakumar Sekar,

Shandong University, China

Ramachandran Vinayagam, Yeungnam University, South Korea

*Correspondence:

Selvaraj Kunjiappan

selvapharmabio@gmail.com

Murugesan Sankaranarayanan murugesan@pilani.bits-pilani.ac.in

Specialty section:

This article was submitted to Nanomaterials,

a section of the journal

Frontiers in Nanotechnology

Received: 07 February 2021 Accepted: 09 June 2021

Published: 23 June 2021

Citation:

Pandian SRK, Panneerselvam T, Pavadai P, Govindaraj S,

Ravishankar V, Palanisamy $P$,

Sampath M, Sankaranarayanan M and

Kunjiappan S (2021) Nano Based Approach for the Treatment of

Neglected Tropical Diseases.

Front. Nanotechnol. 3:665274.

doi: 10.3389/fnano.2021.665274
Neglected tropical diseases (NTDs) afflict more than one billion peoples in the world's poorest countries. The World Health Organization (WHO) has recorded seventeen NTDs in its portfolio, mainly caused by bacterial, protozoal, parasitic, and viral infections. Each of the NTDs has its unique challenges on human health such as interventions for control, prevention, diagnosis, and treatment. Research for the development of new drug molecules against NTDs has not been undertaken by pharmaceutical industries due to high investment and low-returns, which results in limited chemotherapeutics in the market. In addition, conventional chemotherapies for the treatment of NTDs are unsatisfactory due to its low efficacy, increased drug resistance, short half-life, potential or harmful fatal toxic side effects, and drug incompetence to reach the site of parasite infection. In this context, active chemotherapies are considered to be re-formulated by overcoming these toxic side effects via a tissue-specific targeted drug delivery system. This review mainly emphasizes the recent developments of nanomaterial-based drug delivery systems for the effective treatment of NTDs especially sleeping sickness, leishmaniasis, chagas disease, soil-transmitted helminthiasis, african trypanosomiasis and dengue. Nanomaterials based drug delivery systems offer enhanced and effective alternative therapy through the re-formulation approach of conventional drugs into site-specific targeted delivery of drugs.

Keywords: nanomaterials, drug delivery, sleeping sickness, leishmaniasis, chagas disease, soil-transmitted helminthiasis, African trypanosomiasis

\section{INTRODUCTION}

Neglected tropical diseases (NTDs) are diseases endemic to 149 countries in tropical and sub-tropical climatic conditions (Garchitorena et al., 2017). NTDs mainly afflicted peoples in the poorest countries with little visibility of low political voice, without adequate sanitation, limited clean water \& healthcare. Also, people's lives in close contact with infectious vectors, domestic animals and 


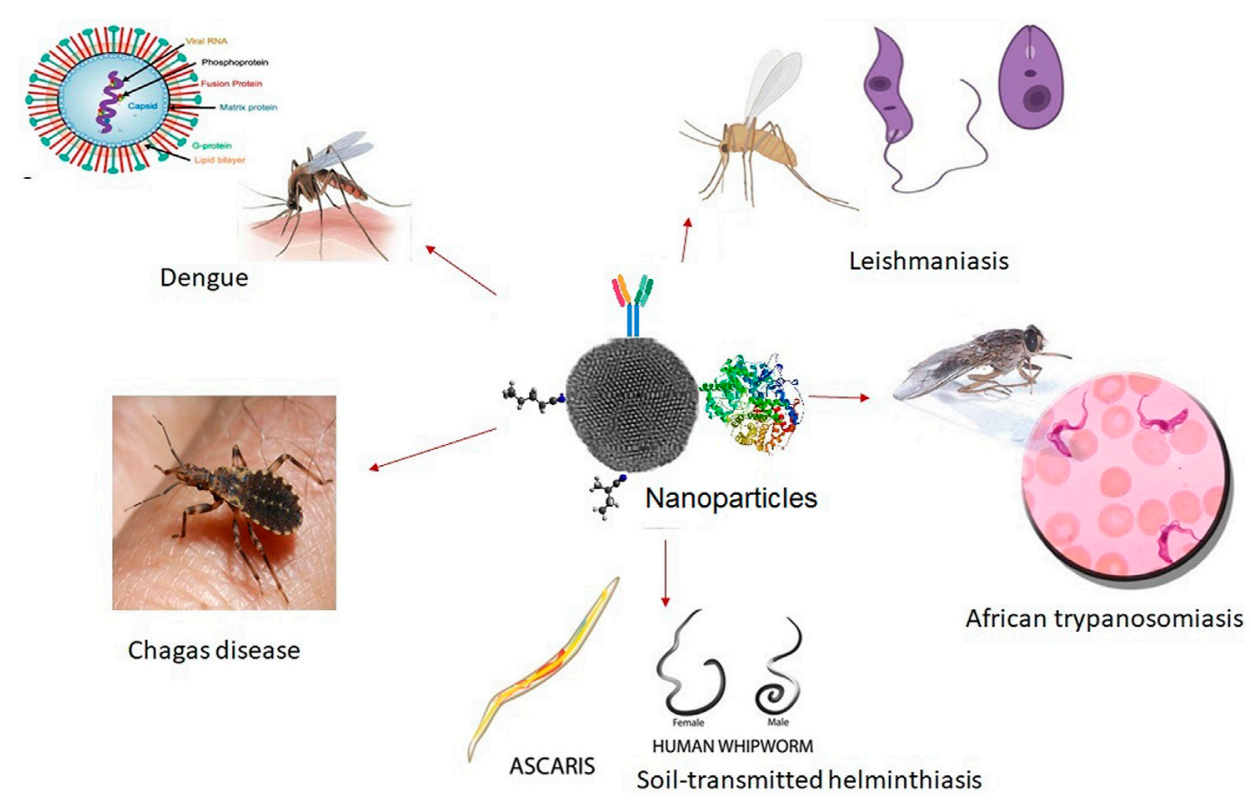

GRAPHICAL ABSTRACT |

livestock (Sun and Amon, 2018). These diseases not only affect health, but also disproportionately to their economic prosperity of the poorest and marginalized communities living in the regions of Africa, Asia and Latin America (World Health Organization, 2015). Globally, about 2 billion peoples are at risk of one or more NTDs and more than 1 billion peoples are affected by these diseases (Mitra and Mawson, 2017). It is estimated that due to NTDs, 57 million disability adjusted life years (DALYs) are lost annually. Recent surveys of the morbidity and mortality caused by these diseases indicate that the burden of DALYs may be higher than reported (Kassebaum et al., 2016). Moreover, NTDs not only cause death but may also have effects for long-term disabilities and diseases, such as exhaustion, blindness, disfigurement, diminished childhood intellectual, physical and cognitive development, as well as reduced productivity of workers, among other conditions (Levine, 2007). They are also rooted to cause various clinical difficulties, such as anemia and other forms of malnutrition (Mackey et al., 2014).

The World Health Organization (WHO) has recorded seventeen NTDs in its portfolio, and these are mainly caused by bacterial, protozoal, parasitic and viral infections such as african trypanosomiasis (sleeping sickness), buruli ulcer, dengue, dracunculiasis, echinococcosis, foodborne trematodiasis, human leishmaniasis, chagas, leprosy, lymphatic filariasis (elephantiasis), onchocerciasis (river blindness), rabies, schistosomiasis (snail fever), soil-borne helminthiasis (intestinal worms), taeniasis/cysticercosis (pig tapeworm), etc (World Health Organization, 2010). In order to improve the health and socioeconomic benefits of the poorest communities, the global reaction of NTDs was therefore appropriate. In recent years, despite the monitoring and removal of NTDs, the participation from the international community and support from governmental or non-governmental donors has been an important driving force (Molyneux and Malecela, 2011). In the year 2018, WHO has welcomed the declaration of new funding to sustain global efforts to eliminate NTDs and avert the devastating impact they cause to mainly poor populations across the globe (Weng et al., 2018).

Furthermore, to accelerate the prevention, monitoring, elimination and eradication of NTDs, the WHO recommends five public-health interventions: preventive chemotherapy, intensified case management, vector control, veterinary public health and clean water, sanitation and hygiene (World Health Organization, 2013). While one approach can dominate the control of a particular disease or a group of diseases, evidence indicates that when all five approaches are combined and administered locally, more successful control can occurs. Currently, research has been taken place in all five interventions; however, several of these NTDs can be controlled with a mass treatment using chemotherapeutics (Lustigman et al., 2012). For instance, rifampicin and streptomycin (buruli ulcer) (Phillips et al., 2020), benznidazole and nifurtimox (chagas disease) (Jackson et al., 2020), acetaminophen (dengue) (Ferreira et al., 2020), metronidazole or thiabendazole (dracunculiasis) (Horton, 2017), albendazole (echinococcosis) (Darvishi et al., 2020), amphotericin B (leishmaniasis) (Berenguer et al., 2020), rifampicin, clofazimine and dapsone (leprosy) (Abdelmaksoud and Gupta, 2020). It is really important that people who are affected by any of the NTDs are handled exactly as prescribed, finish the prescription, and take the complete medications. They may become sick again if they stop the drugs too soon; if they do not take the drugs properly because the causative bacteria, protozoa or virus of NTDs still alive inside the host body may become resistant to those drugs. 
TABLE 1 | Nanoparticle based therapy in treating NTDs.

\begin{tabular}{|c|c|c|c|c|c|}
\hline $\begin{array}{l}\text { S. } \\
\text { No }\end{array}$ & Nanoparticle & Type & $\begin{array}{l}\text { Drug or } \\
\text { mode }\end{array}$ & Disease & References \\
\hline 1. & Gold NPs & Metal & Quercetin & Leishmaniasis & Das et al. (2013) \\
\hline 2. & & & $\begin{array}{l}\text { Couroupita guianensis reduced } \\
\text { Au NPs }\end{array}$ & Malaria larvae & Subramaniam et al. (2016) \\
\hline 3. & & & Kaempferol & Leishmaniasis & Halder et al. (2017) \\
\hline 4. & & & Ciprofloxacin & Plasmodium falciparum & $\begin{array}{l}\text { Varela-Aramburu et al. } \\
\text { (2020) }\end{array}$ \\
\hline 5. & & & MSP10 Oligonucleotides & Plasmodium vivax in urine diagnosis & Alnasser et al. (2016) \\
\hline 6. & & & Thermotherapy & Leishmaniasis & Sazgarnia et al. (2013) \\
\hline 7. & Silver NPs & & & Giardia lamblia cysts & Said et al. (2012) \\
\hline 8. & & & & Leishmaniasis & Allahverdiyev A. et al. (2011) \\
\hline 9. & & & & $\begin{array}{l}\text { Entamoeba histolytica and } \\
\text { Cryptosporidium parvum }\end{array}$ & Saad et al. (2015) \\
\hline 10. & & & Catharanthus roseus extract & Plasmodium falciparum & Ponarulselvam et al. (2012) \\
\hline 11. & & & & Fascioliasis & Gherbawy et al. (2013) \\
\hline 12. & $\mathrm{Ag}_{2} \mathrm{O}$ & & & Leishmaniasis & $\begin{array}{l}\text { Allahverdiyev A. M. et al. } \\
\text { (2011) }\end{array}$ \\
\hline 13. & Copper (II) nano hybrid solids & & & Inhibition of plasmepsin II & Mohapatra et al. (2010) \\
\hline 14. & Copper Oxide NPs & & & $\begin{array}{l}\text { Entamoeba histolytica and } \\
\text { Cryptosporidium parvum }\end{array}$ & Saad et al. (2015) \\
\hline 15. & $\mathrm{TiO}_{2} \mathrm{NPs}$ & & & Leishmaniasis & $\begin{array}{l}\text { Allahverdiyev A. M. et al. } \\
\text { (2011) }\end{array}$ \\
\hline 16. & Magnetic solid lipid NPs & $\begin{array}{l}\text { Metal and } \\
\text { Organic NPs }\end{array}$ & Albendazole & & Abidi et al. (2018) \\
\hline 17. & $\begin{array}{l}\text { Andrographolide } \\
\text { nanoparticles }\end{array}$ & Organic & Andrographolide & Leishmaniasis & Roy et al. (2010) \\
\hline 18. & Chitosan NPs & & S-nitroso-mercaptosuccinic acid & Leishmaniasis & Cabral et al. (2019) \\
\hline 19. & & & Siparuna guianensis essential oil & Aedes aegypti larvae & Ferreira et al. (2019) \\
\hline 20. & & & & Giardia lamblia cysts & Said et al. (2012) \\
\hline 21. & & & Chloroquine & Plasmodium berghei & Tripathy et al. (2012) \\
\hline 22. & & & Albendazole & Echinococcus multilocularis & Abulaihaiti et al. (2015) \\
\hline 23. & Albumin based NPs & & NS1 antibody & Dengue fever diagnosis & Linares et al. (2013) \\
\hline 24. & Abiotic hydrogel nanoparticle & & & Dermonecrosis & O'brien et al. (2018) \\
\hline 25. & Beeswax-Copaiba oil NPs & & Diethyldithio carbamate & Leishmaniasis & Mazur et al. (2019) \\
\hline 26. & $\begin{array}{l}\text { Poly(D, L -lactide-co- } \\
\text { glycolide) NPs }\end{array}$ & & Amphotericin B & Leishmaniasis & Venier-Julienne et al. (1995) \\
\hline 27. & Lipid nanoparticles & & Curcuminoid & Malaria & Nayak et al. (2010) \\
\hline 28. & Liposome & & Amphotericin B & Leishmaniasis & Saravolatz et al. (2006) \\
\hline 29. & & & Andrographolide & Leishmaniasis & Sinha et al. (2000) \\
\hline 30. & Solid lipid NPs & & $\begin{array}{l}\text { S-benzyl dithiocarbazate } \\
\text { (H2bdtc) }\end{array}$ & Trypanosoma cruzi & Carneiro et al. (2014) \\
\hline
\end{tabular}

Drug resistant bacteria, protozoa, and viruses are harder and more difficult to handle once if they acquired to develop (Bank, 2017).

Despite the major developments achieved in medicinal research during the past 50 years, but pharmaceutical industries have not undertaken any kind of research for the development of new medicine against NTDs due to high investment and low-returns; which results in limited chemotherapeutics available in the market (Njoroge et al., 2014). On the other side, the conventional drugs are unsatisfactory due to low efficacy, increased drug resistance, short half-life, potential or harmful fatal toxic side effects, and drug incompetence to reach the site of parasite infection. In particular, drug resistance (the decline in drug efficiency) is a major challenge to public health, and its effect has the potential to kill millions of people around the world (Mondal et al., 2010). It is also an important commercial obstacle for pharmaceutical firms in the private sector since new drugs against NTDs are always costly and require long-term proposals. In this context, conventional drugs are considered to be re-formulated by overcoming these toxic side effects via tissue-specific targeted drug delivery systems through nanoscience/nanotechnology procedures may find new avenues. However, several other diseases including cancer (Hossen et al., 2019), diabetes (Selvaraj et al., 2017), alzheimer's (Oesterling et al., 2014), cardiovascular complications (atherosclerosis) (Gupta et al., 2019), etc., were successfully controlled by re-formulation of conventional drugs into nanomedicine (Chowdhury et al., 2017) has been very well reported by several researchers across the world.

Nanocarrier based novel drug delivery system (NDDS) or nanomedicine is an emerging field that works on nanosized particles for the treatment of chronic human diseases, diagnosis and tissue regeneration. For instance, nanotechnology enabled materials have been created or manipulated for the delivery of therapeutic agents to a disease 
site in a controlled or sustainable release manner as well as diagnosis of various complications, like cancer, diabetes mellitus, osteoarthritis, cardiovascular, tropical infectious diseases, etc., (Chowdhury et al., 2017). The combined research works of nanotechnology and pharmaceutical sciences are encouraging and has grown up very rapidly in recent years. As nano-engineering enabled drug delivery materials intended at the atomic or molecular level, they are usually nanoscaled size. Thus, they can freely circulate the entire human body as compared to bigger materials or conventional drugs. In addition, special structural, chemical, mechanical, magnetic, electrical, and biological properties are shown by nanoscale-sized particles (Zhang et al., 2018). Importantly, NDDS can boost drug stability and water solubility, increase circulation time, promote drug uptake/entry into target cells or tissues, and decrease enzyme degradation, thus increases drug safety and efficacy (Biffi et al., 2019). In addition, for improved bioavailability, NDDS can be delivered by different methods, including inhalation, topical, oral, or intravenous injection. A wide variety of nano-engineering enabled drug delivery materials have already been approved, or are currently undergoing the approval process, to be used for the treatment of NTDs. Table 1 Nanoparticle based therapy in treating NTDs. In recent years, many university research centers and pharmaceutical industries have started to design and develop NDDS for the diagnosis and therapy of NTDs (Long et al., 2015). The recent advances in nanomaterial-based drug delivery systems for the successful treatment of NTDs, particularly leishmaniasis (Gour et al., 2009), chagas disease (Morilla and Romero, 2015), soiltransmitted helminthiasis (Hoekendijk et al., 2016), african trypanosomiasis/sleeping sickness (Stijlemans et al., 2017) and dengue (Silva et al., 2012; Pinto et al., 2021) are mainly highlighted in this study. Finally, we examined potential approaches to improve research and development of economic nano-engineered materials for NTDs to facilitate their translation into clinics and social implementation.

\section{NEGLECTED TROPICAL DISEASES}

\section{Leishmaniasis}

Leishmaniasis is one of the NTDs caused by the pathogenic parasite leishmania species (Protozoa, Trypanosomatidae) (Vermelho et al., 2017). This parasite was carried by the sand flies (mainly female Phlebotomus and Lutzomyia) and transmitted to human beings by the bite of infected sand flies. Sandflies are bred in moist soil, forest areas, caves or the tunnels of rodents and feed from infected animal reservoir hosts or humans. Via a blood transfusion or shared needles, humans may also spread the parasite within themselves (Katz et al., 1989). Transmission can also take place from human to sand fly to human in certain parts of the world. Globally, 1.5 to 2 million new cases recorded each year, 350 million are at risk of acquiring the disease, and leishmaniasis causes 70,000 deaths per year (Torres-Guerrero et al., 2017). In the year 2017, 20,792 out of 22,145 (94\%) new cases reported to WHO occurred in seven countries: Brazil, Ethiopia, India, Kenya, Somalia, South
Sudan and Sudan (World Health Organization, 2018). Brazil accounts for $96 \%(57,582)$ of these cases. The incidence rate of VL increased from 1990 to 2018 by $52.9 \%$ in this country alone with an even higher increase in children under the age of 1 year (Ehrenberg et al., 2021). Leishmaniasis is correlated with malnutrition, population relocation, inadequate housing, weak immune system, lack of financial support, and primarily affects some of the poorest people on earth. Leishmaniasis is a diverse group of diseases with a wide variety of clinical symptoms, including self-healing cutaneous leishmaniasis [caused by leishmania in the old world (the Eastern Hemisphere), include Leishmania tropica, L. major, L. ethiopica], mucosal leishmaniasis [it is caused by new world (American) leishmania spp. such as $L$. braziliensis] and systemic visceral leishmaniasis (it is caused by $L$. donovani/L. infantum), the infection can range from asymptomatic to severe fatal (Rock et al., 2016).

The most common type of leishmaniasis is cutaneous leishmaniasis (CL), which causes disfiguring skin lesions that can leave life-long scars especially for women and children, lead to severe disability or stigma (Al-Kamel, 2017). In the Americas, the Mediterranean basin, the Middle East and Central Asia, about $95 \%$ of CL cases occur. Over $85 \%$ of new CL cases occurred in 10 countries in the year 2018, the WHO reported: Afghanistan, Algeria, Bolivia, Brazil, Colombia, Iran (the Islamic Republic of), Iraq, Pakistan, the Syrian Arab Republic, and Tunisia. Around 600,000 to 1 million new cases are expected to occur annually worldwide (Nweze et al., 2020). Mucosal leishmaniasis (ML) causes the mucous membranes of the nose, mouth and throat to become partially or completely destroyed (Amato et al., 2008). In Bolivia (Plurinational State of), Brazil, Ethiopia and Peru, over 90\% of mucosal leishmaniasis cases occur (Ahmed et al., 2018). Serological tests have limited validity for both CL and ML, and the diagnosis is validated by clinical manifestation using serological tests. CL and ML can contribute to severe morbidity, while visceral leishmaniasis (VL) (also known as Kala-azar and black fever) can lead to death if left untreated in 95\% of instances (Nagle et al., 2014). Irregular episodes of fever, weight loss, enlargement of spleen, liver, and anemia are characterized by VL (Garg and Dube, 2006). In Brazil, East Africa and India, most cases occur. Each year, an estimated 50,000-90,000 new VL cases occur globally, with only $25-45 \%$ reported to the WHO. It ranks second among NTDs in mortality and fourth in morbidity (NTDs). In the year 2018, more than 95\% of new cases reported to WHO occurred in 10 countries: Brazil, China, Ethiopia, India, Iraq, Kenya, Nepal, Somalia, South Sudan and Sudan (Mitra and Mawson, 2017).

\section{Current Challenges in the Treatment of Leishmaniasis Infection}

A satisfactory vaccine is not yet established against leishmaniasis although it seems to be very effective for controlling the leishmaniasis. At present, the control/treatment measures for leishmaniasis are challenging and there are systemic and local treatments, like chemotherapy and/or non-pharmaceutical methods are available (Wijerathna et al., 2017). As compared to all other NTDs, leishmaniasis has suffered a historical lack of attention by pharmaceutical industries, largely because they 
thrive in poverty conditions (Kealey, 2010). Thus, the development of vaccine cannot be expected in the near future as well. Hence, control measures rely only on chemotherapy to alleviate the disease and on vector control to reduce transmission. In the current situation, few drugs have emerged for the treatment of leishmaniasis, namely, pentavalent antimonials (gold standard drug for the treatment of leishmaniasis), amphotericin $\mathrm{B}$, meglumine antimoniate, sodium stibogluconate, sitamaqine, miltefosine, paromomycin, pentamidine, imiquimod and azoles (ketoconazole, fluconazole, itraconazole). These drugs are used as a combination therapy to enhance the efficacy and reduce the toxicity (Kulshrestha et al., 2012). At the same time, none of these available chemotherapies can be considered suitable due to their high toxicity, drug resistance against parasite, long duration of treatment, and severe adverse reaction, leads to withdrawn the course of drug treatment. Moreover, these drugs have been associated with hypoglycaemia, nephrotoxicity, hepotoxicity, hypotension, and cardiopathy (Capela et al., 2019).

\section{Chagas Disease}

A neglected parasitic infection caused by the protozoan Trypanosoma cruzi is chagas disease or American trypanosomiasis (Rassi and De Rezende, 2012). Owing to its high mortality and morbidity rates, it is one of the most significant public health issues in Latin America (Dias et al., 2002). Chagas disease also presents in non-endemic countries, such as United States of America (United States), Canada, Europe, Japan, and Australia (Gascon et al., 2010). Around the globe, nearly 6-7 million people are infected by $T$. cruzi parasite that causes chagas disease (Schmunis, 1991). T. cruzi is mainly transmitted to humans by feces/urine contamination of the bloodsucking triatomine (reduviid) bugs. The parasite may also be transferred during pregnancy from an infected woman to her infant (more than one-quarter of the world's new cases of chagas disease), tainted blood transfusion, organ transplantation, transplacental transmission, and foodborne transmission (vectorcontaminated food, drinks and feces) (Roellig et al., 2009). The $T$. cruzi biological cycle includes: the invertebrate vector (triatomine insects) and the vertebrate host (human) are two intermediate hosts and have three fundamental types, namely, trypomastigotes, amastigotes and epimastigotes. 1) trypomastigotes (An infected triatomine bug) takes a blood meal and releases trypomastigotes in its feces near the bite wound site. Via the bite wound or intact mucosal membranes, trypomastigotes join the host (the amastigotes multiply by binary fission inside mammalian host cells and differentiate into trypomastigotes, and then are released into the circulation as bloodstream trypomastigotes). 2) the amastigotes reproduce by binary fission and differentiate into trypomastigotes, and then are released into the circulation as blood trypomastigotes. Trypomastigotes infect cells from a spread of tissues and transform into intracellular amastigotes in new infection sites. Clinical manifestations may end up from this infective cycle. The bloodstream trypomastigotes don't replicate (different from the African trypanosomes). Replication restarts only the parasites enter another cell or are eaten by another vector. The "kissing bug" becomes infected by feeding on human or animal blood that contains current parasites. 3) the eaten trypomastigotes transform into epimastigotes with in the vector's midgut. The parasites reproduce and differentiate in the midgut and differentiate into infective metacyclic trypomastigotes in the hindgut (Onyekwelu, 2019).

A sudden, brief illness (acute) may cause chagas disease, or it may be a long-lasting (chronic) condition. Symptoms may vary from mild to extreme, but usually no symptoms are seen among many affected individuals, most are unaware that they are sick. Up to one third of infected individuals will experience heart damage, which can lead to progressive heart failure or premature death, becoming noticeable only several years and even decades later. Chagas disease is an extreme opportunistic infection that results in meningoencephalitis and myocarditis in people living with HIV/AIDS (Bustamante et al., 2014; Jansen et al., 2015).

\section{Current challenges in the treatment of Chagas disease} Chagas disease was discovered by the Brazilian sanitary physician and researcher Carlos Justiniano Ribeiro Chagas 110 years ago (Lidani et al., 2019). Still, there is no effective treatment or reliable vaccine for this disease. In the late 1960s and early 1970s two nitroheterocyclic drugs, namely, 5-nitrofuran nifurtimox (NFX) and 2-nitroimidazole benznidazole (BNZ) respectively, were discovered especially for the treatment of chagas disease (Junior et al., 2017). The drugs (NFX and BNZ) are approved by the US FDA and both of them are contraindicated in pregnant women (Morilla and Romero, 2015). When treatment is started at the beginning of infection and during the acute stage of the disease, both medications are highly successful (up to $80 \%$ of parasitological cures). However, in the acute stage, very few patients are diagnosed because many persons are asymptomatic when they are first infected or because patients do not have access to adequate diagnosis and care (De Figueiredo Diniz et al., 2013). Moreover, the currently available drugs are of low bioavailable at the site of infection with drug resistance; in long-term therapy drugs cause serious toxic side effects (Apt, 2010). Up to now, several research works have been conducted for vaccine development against T. cruzi infection, a safe and effective vaccine for human use is not available, ranging from parasite extracts to genetically engineered parasites, and while some demonstrated promising efficacy in animal models (ArceFonseca et al., 2015). A defensive antigen and the production of attenuated parasites that will not activate pathology in the long term have been the key problems observed. Therefore, management strategies for chagas disease remain incomplete for treatment in the absence of vaccine or successful drug candidates.

\section{Soil-Transmitted Helminthiasis}

Soil-transmitted helminthiasis (STHs) denotes the intestinal nematodes infecting humans that are transmitted through contaminated soil (Mascarini-Serra, 2011). An estimated 1.5 billion people are infected with STHs and more than $24 \%$ of the world's population lives in places endemic for STHs (Hotez et al., 2006). Infections are widely distributed in tropical and subtropical areas, with the greatest numbers occurred in sub-Saharan Africa, America, China, and East Asia (De Silva et al., 2003). STHs 
are transmitted by eggs that are passed in the feces of infected people. Infections with STHs are found mainly in people living in areas with warm and moist climates, where lack of adequate sanitation, water and hygienic. These infected peoples are at higher risk of disease and are also the main source of environmental contamination (Mascarini-Serra, 2011). At worldwide, the STHs are infected by roundworms (approximately 807-1,121 million peoples infected with Ascaris lumbricoides), whipworms (approximately 604-795 million peoples infected with Trichuris trichiura), and hookworm (approximately 576-740 million peoples infected with Ancylostoma duodenale and Necator americanus) that live for years in the human gastrointestinal tract, where worms feed on host tissues, including blood, which leads to a loss of protein and minerals. These can be serious concerns such as anemia, reduction of nutritional intake, impaired cognitive and physical fitness in children (Darlan et al., 2017). Also, these helminth infections normally do not cause mortality, however, they are chronic in condition can lead to significant morbidity including blindness, organ damage, physical disabilities (Finlay et al., 2014). In the year 2017, the global burden of STHs infection was estimated as 1.9 million disability adjusted life years (DALYs) (Martins-Melo et al., 2018). The adult worms inhabit in the host gastrointestinal tract, where they produce thousands of eggs each day that are passed in the feces. The eggs grow into infectious stages whenever the environmental conditions are favorable. Infection can occurs when infected eggs (Ascaris lumbricoides and Trichuris trichiura) or larvae (Ancylostoma duodenale) are consumed in contaminated foods (e.g., vegetables that are not carefully cooked, washed or peeled), hands or utensils, or when contagious hookworm larvae penetrate the skin from contaminated soil (Necator americanus and Ancylostoma duodenale). There is no immediate person-to-person transmission or contamination from new feces since it takes around 3 weeks for eggs passing into feces to become infectious in the soil. The worms do not replicate inside the host, which is a significant feature of these parasites' epidemiology (Else et al., 2020).

\section{Current Challenges in the Treatment of STHs Infection}

The WHO's goal is to reduce the incidence of moderate and serious STHs infections in pre-school and school-aged children to below $1 \%$ by the year 2020 (Becker et al., 2018). School-aged children in endemic areas are enrolled routinely in so-called preventive chemotherapy services to accomplish this purpose. Currently, chemotherapeutic for STHs infections are limited to a minimal set of drugs; these include albendazole (ABZ), mebendazole (MBZ), levamisole, niclosamide, praziquantel (PZQ), pyrantel pamoate and ivermectin. Apart from their different activities, these drugs share a common set of limitations (Conterno et al., 2020).

These available drugs are orally administrated and has poor water solubility which results in extensive first pass metabolism and leads to low bio-availability. Benzimidazole drugs (ABZ and MBZ) are, for example, a wide range of anthelmintic action that is effective in regulating a variety of species of nematodes and cestodes (Holden-Dye and Walker, 2014). Inhibiting tubulin polymerization, which results in cytoplasmic microtubule degradation is the key mode of action. Benzimidazole drugs are lipophilic with poor solublility in water, which undergoes extensive first pass metabolism. Also, it has poorly absorbed through gastrointestinal tract with less oral bioavailability $(10 \%)$ and lesser half-life in patients with normal hepatic function. Likewise, ivermectin has a solubility of only $4 \mu \mathrm{g} \mathrm{ml}^{-1}$ in water and increases the solubility by addition of surfactants and administrated in the form of micelles (Gottschall et al., 1990). Also, these drugs have a short circulation time and fast washout from the blood plasma. For instance, the effective plasma half-life of PZQ is less than $2 \mathrm{~h}$, with $4-6 \mathrm{~h}$ half-life for its active metabolic derivatives (Andrews et al., 1983). Thus, the drug does not accumulate/reach the site of infection even if repeated doses are administered. Moreover, the available chemotherapeutics often exhibit systemic toxicity. For instance, $A B Z$ and MBZ may cause adverse effects, such as transient gastrointestinal discomfort, headache, and leukopenia. Although, several potential medications, including severe neuropsychiatric illnesses, damage to reproductive organs, and even death, cause significant side effects in humans at therapeutic doses (Hong, 2018). In the view of insufficient efficacy, potential drug resistance against pathogens from longterm use, there is not only a pressing need for the development of new treatments against STHs infections but also a need to optimize current treatment schemes.

\section{Human African Trypanosomiasis}

Human African trypanosomiasis or African trypanosomiasis, also known as sleeping sickness, is a vector-borne parasitic disease (Malvy and Chappuis, 2011). The incidence of HAT reported cases found in sub-Saharan Africa regions between the latitudes of $14^{\circ}$ north and $29^{\circ}$ south (Lindoso and Lindoso, 2009). About 60 million people worldwide are at risk of developing the disease. Trypanosomiasis is caused by infection with protozoan parasites of the Trypanosoma genus belonging to Trypanosoma brucei, which affects both human population and animals. Bites of the blood sucking tsetse flies (genus Glossina) who have acquired their infection from humans or animals harboring human pathogenic parasites are being transmitted to humans (Radwanska et al., 2018). There are two clinical isoforms of HAT, based on the infective parasites involved (Kennedy, 2013). T. brucei gambiense causes a slowly progressing African trypanosomiasis, it is present in around 24 countries in the west and central Africa. This forms are more common and causes about $98 \%$ of reported cases of sleeping sickness and produces chronic infection. T. brucei rhodesiense, on the other hand, is present in around 13 eastern and southern African countries. This form currently accounts for less than $2 \%$ of confirmed cases and triggers an acute infection that can develop quickly to affect the nervous system. In addition, HAT occurs in two sequential stages, often followed by the growth of a trypanosomal chancre (indurated red or purple nodule, $2-5 \mathrm{~cm}$ in diameter, which is accompanied by enlarged lymph nodes) at the inoculation site within days of an infected fly being bitten. The hemolymphatic system, in which parasites are replicated in the blood and lymph, is an early stage of 
HAT infection. The first stage include signs and symptoms that are not clear, such as intermittent fever, pruritus, and lymphadenopathy. A late stage of HAT infection is a meningo-encephalitic or neurological stage, with severe signs of central nervous system (CNS) involvement when parasites are supposed to have crossed the blood-brain barrier. Generally speaking, this is when there are more apparent signs and symptoms of the disease: personality changes, confusion, sensory problems and impaired coordination. A significant characteristic is the interruption of the sleep cycle, which gives the disease its name. Sleeping sickness is deemed fatal without treatment, although cases of stable carriers have been recorded (Brun et al., 2010).

\section{Current Challenges in the Treatment of HAT Infection}

There is no immunization available against HAT and vaccine is impossible that will be created sooner rather than later. The main reason is the ability of the parasite to escape antigenic variety from the host immune system, altering the significant surface glycoprotein present on its surface to prevent antibody-mediated reactions (Lightowlers et al., 2003). While, few drugs were approved for the treatment against HAT based on form of the disease stage and the infecting sub-species. In the first or early stage of T. brucei, pentamidine and suramin are used as first line agents but the drug pentamidine is quit toxic. For the second stage of gambiense, melarsoprol, eflornithine has been used. These drugs are discovered between 1920-1949 and the mode of action of these drugs is still poorly understood. Another drug, nifurtimox (introduced in the year 2009), is used in combination under special authorizations (Ebikeme, 2007). A newer drug, fexinidazole is an orally administred drug used in the treatment of both stages of T. brucei gambiense (Lindner et al., 2020). These trypanocidal drugs are unsatisfactory for a number of reasons, including severe toxic side effects. Neither pharmaceutical industries nor government in endemic nations have indicated interest to improve the helpful choices accessible for the treatment (Barrett et al., 2007). Therefore, in the year 1975, WHO listed trypanosomiasis as one of the neglected diseases to encourage the endemic states to develop more effective drugs. Still there is no new drugs identified and approved for the treatment of HAT (Chatelain and Ioset, 2011).

\section{Dengue}

Dengue fever is a viral infection transmitted by female mosquitos that is a major health concern in the tropical and sub-tropical regions between $30^{\circ} \mathrm{N}$ and $40^{\circ} \mathrm{S}$, with the potential to spread to other parts of the world (Mulyatno et al., 2012; Volpedo et al., 2019). Dengue fever is currently widespread in over 100 countries in Africa, the Americas, Southeast Asia, the Western Pacific, and the eastern Mediterranean (Nathan and Dayal-Drager, 2006). According to study published in the year 2019 on the present and potential global spread of dengue virus, approximately $53 \%$ of the world's population (between 3.45 and 4.09 billion people) is at risk of infection (Messina et al., 2019). Female mosquitos of the species Aedes egypti and, to a lesser degree, Aedes albopictus, which breed in the peridomestic climate, transmit the dengu virus (Bonizzoni et al., 2013). Chikungunya and Zika viruses are also transmitted by these mosquitos (Magalhaes et al., 2018). Dengue virus is a single-stranded enveloped RNA genome that belongs to the Flaviviridae family (genus: Flavivrus). There are four serotypes of the virus that cause dengue fever (DENV-1, DENV-2, DENV-3 and DENV-4) (Weaver and Vasilakis, 2009). When you are infected with one form of dengue virus, you are immune to that virus for the rest of your life (Murphy and Whitehead, 2011). A individual will develop viremia, or a high level of the dengue virus in the blood, four days after being bitten by an infected mosquito. Sudden onset of high fever, extreme headache and retro-orbital pain, myalgia, arthralgia, a maculopapular rash, and mild hemorrhage fever are all common clinical symptoms (Chowdhury et al., 2012).

\section{Current Challenges in the Treatment of Dengue}

There is no appropriate cure, therapy, or vaccine for dengue/ severe dengue as of yet. Early identification of the disease progression associated with severe dengue, as well as access to adequate medical treatment, decreases severe dengue fatality rates to less than $1 \%$. The case fatality rate in serious dengue infection may be as high as $5-10 \%$ if it is not recognized early or treated (Rigau-Pérez, 2006). Treatment with immunomodulators such as chloroquine or statins is recommended since dengue infection is marked by the release of multiple inflammatory cytokines (García et al., 2018). Drugs like chloroquine and statins have been repurposed to treat dengue fever. Although, the dengue vaccine (Dengvaxia) is available but it showed high risk of causing serious dengue infection after vaccination, particularly in children (Forsyth, 2020). Medicinal compounds that specifically target the dengue virus by inhibiting viral replication will be the preferred treatment option for dengue infection, but they are currently unavailable. Numerous research groups have already taken bold steps and made significant strides toward developing a vaccine that is safe, affordable, and efficient against all serotypes for the benefit of global public health.

\section{How Nano-Engineered Particles can Improve the Treatment of NTDs}

Recent developments in the field of nanotechnology have proven its efficacy in drug delivery applications and overcoming several limitations of conventional drugs (Chowdhury et al., 2017). The nano-engineering enabled carrier/vehicle can effectively deliver the loaded drugs into the site of infection and eliminate the parasites, viruses, protozoans, etc., from the infected host organisms (Singh et al., 2017). The conventional anti-infective agents/drugs have low tolerability, low bioavailability, longer duration of treatment profile, high level of drug resistance against disease-causing parasites and difficult to administer, significant advantages has been noted in the development of novel nano-biopharmaceuticals that can cure NTDs (Subhaswaraj et al., 2020). The nanoparticles can be designed using materials at the atomic or molecular level, they are typically nano-sized with well-defined physico-chemical and biological properties that may help for further advancements in drug delivery applications (Longmire et al., 2008). Nano-engineering enabled particulate systems to offer several benefits against 
chronic diseases including NTDs (Look et al., 2010). Firstly, these nano-engineered particulate systems can encapsulate a high density of conventional drug molecules that can deliver directly into severely infected specific sites or cells or targetoriented tissues and enhances the bio-availability of drugs. Due to this site specific delivery of drugs that can increase the sensitivity of drugs against disease causing pathogens via stimulation of immune system as well as reduces the drug resistance (Kunjiappan et al., 2020). Secondly, they can circulate freely in the human body as compared to macromolecular conventional drugs. Thirdly, these nanoparticles can be fabricated with a combination of synthetic \& natural polymeric materials that can release the loaded therapeutic molecules in a sustained or controlled manner over several days to months together (Mehtani et al., 2019). The usage of lower therapeutic dose of a conventional drug attached or encapsulated in a nanoengineered particulate system will be advantageous to public health in reducing the toxicity and cost, which had been a significant hindrance in the existing conventional treatment for NTDs (Volpedo et al., 2019). Nanoparticles based drug delivery system include liposomes, solid-lipid nanoparticles (SLNs), nanocrystals, nanostructured lipid carriers (NLC), polymeric micelles, polysaccharide and protein nanoparticles, nanoemulsions, niosomes, inorganic nanoparticles, dendrimers, quantum dots, which have observed significant results in drug delivery against various parasitic infections of NTDs (SantosValle et al., 2019).

\section{Type of Nanoparticles Used for the Treatment of NTDs}

Nanoparticles with the characteristics of excellent biodegradability and bio-compatibility are accounted as the best vehicle for delivering molecules in biomedical applications. The ideal drug delivery system needs to be study their biological and physio-chmical properties. Nanoparticles based drug delivery systems such as solid-lipids, liposomes, dendrimers, noisome, protein, polymeric, and polysaccharide nanoparticles are considered as effective approach for the treatment of chronic human diseases (Duan et al., 2020). Due to their various advantages as a carrier and drug delivery vehicle, solid-lipid nanoparticles (SLNs) have attracted worldwide (Jumaa and Müller, 2000). Phospholipids are important constituents of lipid and lipid-based drug delivery systems due to their amphiphilic nature, bio-compatibility, and multifunctionality. SLNs are commonly spherical shaped with an average diameter of $50-1,000 \mathrm{~nm}$. SLNs are formulated with the ingredients of lipids, emulsifiers, active pharmaceutical ingredients and solvent systems. The preparation of SLNs does not require the need of any organic solvents. The rigid hardcore lipid matrix provides stability and bio-degradability to SLNs. Apart, SLNs provide better entrapment efficiency for hydrophobic drugs, have greater ability of controlled release and targeting ligands. Since, the organic solvents are not required for the synthesis of SLNs, it can provide tremendous reproducibility, feasibility of large-scale production, costeffective, and can adopt during high-pressure homogenization method (Gohla and Dingler, 2001). Unlike polymeric nanomaterials, the lipid core matter of SLNs is physiological and biodegradable which can provide better bio-compatibility and bio-availability. Whereas the polymeric nanomaterials may accumulate unwantedly in the liver, spleen, etc., (Schwarz, 1999).

Liposomes are vital biological nanomaterials that have been reported for various applications over a long period of time. Naturally, they pose nano scale properties, structurally similar to bio-film, excellent bio-compatibility, and sought-after drug delivery systems. Liposomes are highly stable than other polymeric nanomaterials, capable of holding both hydrophilic and hydrophobic molecules, reported in the size range of 5-200 nm (Li et al., 2019). Kidney excretion of drugs can be reduced if the molecules are loaded with liposomes. Furthermore, high content of liposomes can be accumulated at the specific tissues due to their enhanced permeability and retention (EPR) effect (Matsumura and Maeda, 1986). As a natural nanomaterial, liposomes pose following characteristics: cell membrane-like structure, high bio-compatibility and bio-availability, low immunogenicity, ability to protect molecules or active groups from degradation, prolonged drug half-life, and reduced toxicity (Steichen et al., 2013). The use of liposomes has been reported for helminth infections, leishmaniasis (Guery et al., 2017) and protozon diseases (Volpedo et al., 2019).

Niosomes are nano-vesicles comprised of non-ionic surfactants, amphipathic compounds and provides an overall neutral charge. The use of non-ionic surfactants in niosomes made them cost-effective in the applications of biomedicine. Niosomes can carry both hydrophilic and hydrophobic drugs. Niosomes, highly stable and leakier than liposomes are economically alternative to liposomes. Upon freezing, the size of niosomes decreases and subsequent thawing which evidenced by cryo-EM and DLS techniques (Bartelds et al., 2018). The efficacy of niosomes in vitro and in vivo has been extensively reviewed and reported by scientist across the globe (Uchegbu and Vyas, 1998).

Polymeric nanoparticles are made-up of polymers and copolymers with the capability of encapsulating or adsorbing molecules at the inner core or outer surface, respectively. The polymeric nanoparticles are fabricated with a size range of $10-1,000 \mathrm{~nm}$. Polymeric nanoparticles pose core-shell structure; where the inner core consists of polymeric matrix with the capability of holding hydrophobic drug and the surface is mainly made-up of hydrophilic polymers such as polyethylene glycol (PEG) or polyvinyl pyrrolidine (PVP). The surface coating with these materials provides stearic stability, reduced immunogenicity and phagocytosis from reticuloendothelial system. The polymeric nanoparticles are bio-degradable, can enhance bio-availability of drug, pharmacokinetic control, and can reduce immune reactivity against the drug. The use of polymeric nanoparticles in the parasitic disease was extensively reviewed (Date et al., 2007).

Dendrimers are defined as hyper-branched 3D polymeric nanoarchitectures that consist of multiple functional groups on their surface. The compounded functional groups in their surface enhances their functionality and grants them the properties of versatile and bio-compatible. Their unique characteristics such as 
TABLE 2 | The advantages and limitations of various nanocarrier systems.

\begin{tabular}{|c|c|c|c|}
\hline Nanocarrier & Advantages & Limitations & References \\
\hline Liposomes & $\begin{array}{l}\text { Passively target drugs to macrophages, FDA approval in the } \\
\text { treatment of visceral leishmaniasis since 1999, and ability to } \\
\text { carry either hydrophobic or hydrophilic drugs, non- } \\
\text { immunogenic, bio-compatible, bio-degradable, highly stable, } \\
\text { easy enabled surface conjugated with ligands }\end{array}$ & $\begin{array}{l}\text { High production cost, Sometimes phospholipid undergoes } \\
\text { oxidation and hydrolysis-like reactions, leakage and fusion of } \\
\text { encapsulated drug/molecules }\end{array}$ & $\begin{array}{l}\text { Beltrán-Gracia et al. } \\
\text { (2019) }\end{array}$ \\
\hline $\begin{array}{l}\text { Solid-lipid } \\
\text { nanoparticles }\end{array}$ & $\begin{array}{l}\text { Good bio-compatibility, low toxicity and lipophilic drugs are } \\
\text { better delivered by solid-lipid nanoparticles and the system is } \\
\text { physically stable, cost-effective }\end{array}$ & $\begin{array}{l}\text { Low drug-loading capacities, stability problems during } \\
\text { storage or administration, sample dilution or water removal } \\
\text { might significantly change the equilibria between different } \\
\text { colloidal species and physical state of the lipid }\end{array}$ & $\begin{array}{l}\text { Mukherjee et al. } \\
\text { (2009) }\end{array}$ \\
\hline $\begin{array}{l}\text { Polymeric } \\
\text { nanoparticles }\end{array}$ & $\begin{array}{l}\text { Simple to synthesize, higher stability, sustained and extended } \\
\text { drug release profile, bio-compatible, bio-degradable, } \\
\text { nontoxic, prolonged blood circulation period, minimal drug } \\
\text { alteration, less reactive to enzymatic degradation, allow } \\
\text { macrophage-targeted or site-targeted delivery }\end{array}$ & Difficult to scale up & Kamaly et al. (2016) \\
\hline Niosomes & Good stability, low cost, easy to formulate and scaling-up & $\begin{array}{l}\text { Hydrolysis of encapsulated drug which limit the shelf life of the } \\
\text { dispersion }\end{array}$ & $\begin{array}{l}\text { Kumar and Rai } \\
\text { (2011) }\end{array}$ \\
\hline Nanoemulsions & Stable, carry both hydrophobic and lipophilic drugs & $\begin{array}{l}\text { Use of large concentration of surfactant and co-surfactant } \\
\text { necessary for stabilizing the nanodroplets }\end{array}$ & $\begin{array}{l}\text { Patel and Joshi } \\
\text { (2012) }\end{array}$ \\
\hline $\begin{array}{l}\text { Metal } \\
\text { nanoparticles }\end{array}$ & Antibacterial, antifungal properties, stable, uniform structure & Toxicity & Palza (2015) \\
\hline
\end{tabular}

uniformed shape and size, high degree of branching, polyvalency, solubility in water, availability of inner cavities, and uncomplicated synthetic approaches made them sought-after materials for biomedicine and drug delivery applications (Sherje et al., 2018). The dendrimers are differed from other polymeric nanomaterials due to their unique characteristics (Madaan et al., 2014). The toxicity of dendrimers is based on their surface charge and generation dependent (Zinselmeyer et al., 2002). The PAMAM (Poly(amidoamine)) dendrimers pose less toxic than the amino terminated linear polymer. The degree of substitution of amine groups on the surface of dendrimers determines their toxicity. However, the biocompatibility of dendrimers can be enhanced by surface modification with PEG (Frechet and Hawker, 1989).

\section{Nano-Engineered Particles for the Treatment of Leishmaniasis Infection}

It is a major challenge to develop any new drug for the treatment of leishmanial diseases. Recently, nanoscience/nanotechnology has been applied to re-modify the existing anti-leishmanial drugs into nanoparticle-based drug delivery system which is a promising approach for the effective treatment of leishmaniasis (Raj et al., 2020). This nanotechnology-based drug delivery system can offer a great potential to overcome most of the limitations associated with conventional drugs including fatal toxicity, multidrug resistance and most essentially cost factor (Vashist et al., 2016). The Food and Drug Administration (FDA) has recognized various nanoparticulate systems and they are currently used for therapeutic applications (Zhang et al., 2008). Doxil (PEGylated liposomal doxorubicin), for instance, is the first FDA-approved (1995) nano-formulation used to treat some forms of cancers, including metastatic ovarian cancer and Kaposi's sarcoma associated with AIDS (Han et al., 2019). Naturally, good bio-compatibility, effective bio-degradability and nonimmunogenic characteristics of nano-engineered carrier material with conjugated/encapsulated drugs make an ideal candidate for anti-leishmanial therapeutic applications ( $\mathrm{Su}$ and Kang, 2020). In this view, several nano-engineered carrier materials have been used for enhanced delivery of antileishmanial drugs. Among the various nano-engineering enabled carrier systems, polymeric, inorganic, liposomes, and lipid-based nanoparticles are widely used for leishmaniasis treatment, as they are simple and quickly penetrated into macrophages in the liver and spleen. Table $\mathbf{2}$ shows the advantages and limitations of various nanocarrier systems.

Various types of bio-compatible and bio-degradable polymeric materials are used for anti-leishmanial drug delivery applications. Polymeric nanoparticles within the size range from $10-100 \mathrm{~nm}$, can carry active compounds by different strategies like encapsulation/entrapment, chemically conjugated on the surface, dissolution onto the polymeric core. Major advantages of polymeric nanoparticles as drug delivery vehicles include their potential use for controlled release, non-immunogenic, ability to protect the drugs against various body fluids/enzymes, improved bio-availability, allow efficient targeting and therapeutic effect of drugs. Synthetic [polyvinyl alcohol (PVA), polyethylene glycol (PEG), poly (lactic acid) (PLA), poly (cyanoacrylate) (PCA), poly (lactide-co-glycolide) (PLGA), poly (caprolactone) (PCL)] and natural (gelatin, albumin, keratin, alginate, chitosan, hyaluronan, cellulose, silk fibroin, pullulan, collagen and carrageenan) polymers are frequently used for drug delivery applications (Bambole and Yakhmi, 2016). In the assessment of factors like bio-compatibility, bio-degradability, higher drug loading capacity, prolonged circulation, toxic solvent free-formulation, and availability of natural and semi-synthetic polymers are often advantageous as compared to synthetic alternatives. For example, semi-synthetic polycaprolactone biopolymer (PCL) nanoparticles have been synthesized with nanoprecipitation loading of 


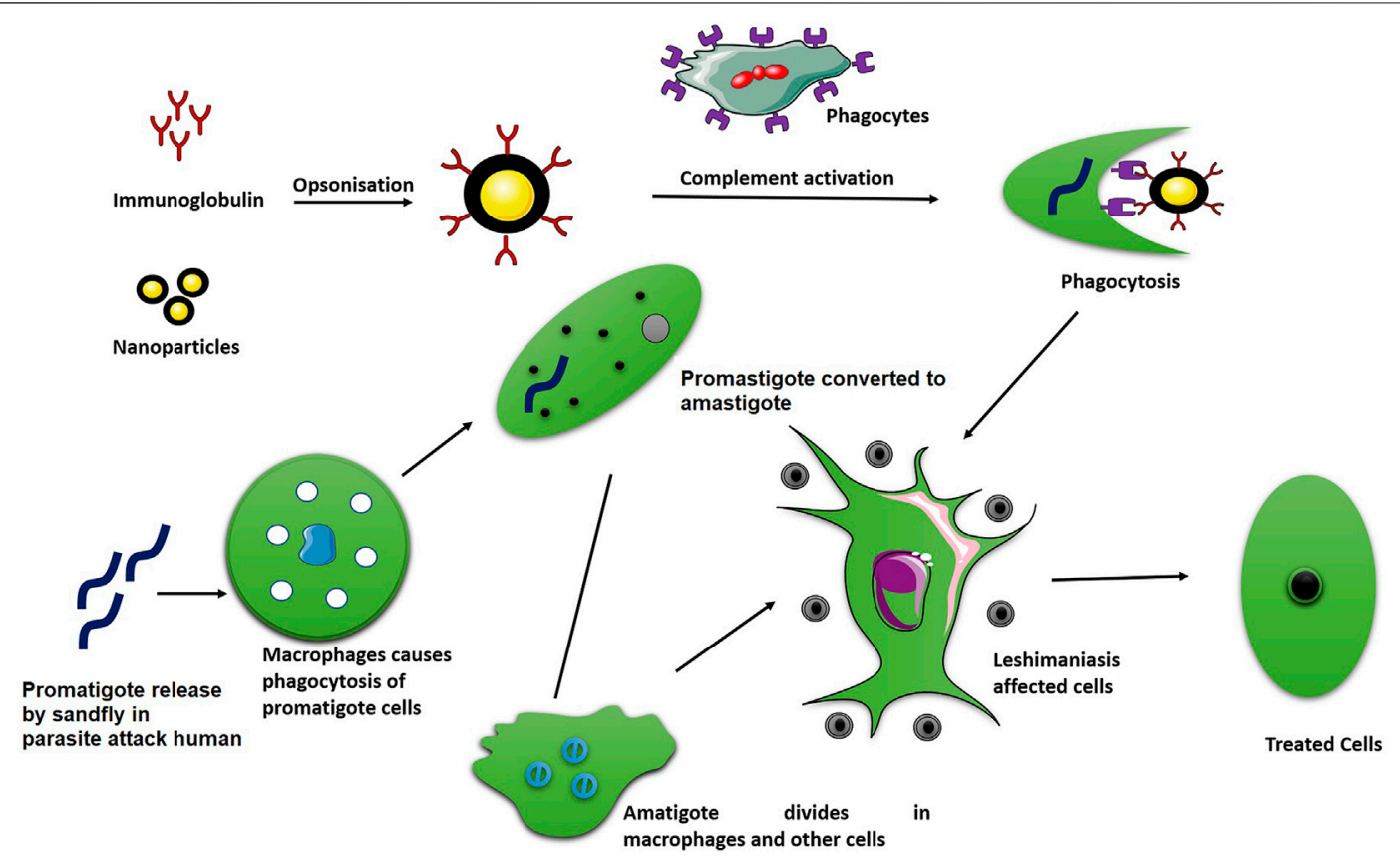

FIGURE 1 | lllustrates the phagocytosis process of absorption of drug loaded nanoparticles.

Amphotericin B for the treatment of topical leishmanial infections (Alquadeib, 2016). For both L. tropica KWH23 and L. donovani amastigotes, the calculated $\mathrm{IC}_{50}$ of the prepared nanoparticle formulation was found to be significantly lower compared to control-free Amphotericin B and AmBisome ${ }^{\circledR}$ to demonstrate maximum parasite inhibition (Saqib et al., 2020). Also, a natural polymer, chitosan loaded with Amphotericin B nanoparticles has been synthesized for the treatment of cutaneous leishmaniasis (CL) (Mehrizi et al., 2019). In vitro activity against L. major intracellular amastigotes was demonstrated by Amphotericin B-loaded nanoparticles with comparable activity to unencapsulated Amphotericin B but significantly lower toxicity to KB-cells and red blood cells (Riezk et al., 2020).

Also, liposomes and lipid-based nanocarrier systems are an excellent choice for the treatment of leishmanial diseases (Date et al., 2007). Efficacy of the drug is not only dependent on its physico-chemical properties, but also the delivery carrier/vehicle system, which could increase the sensitivity of drug, controlled release of drug and enhances the bio-availability of drug with patient safety. In this view, liposomes provides greater stability and efficacy of the active anti-leishmanial drug, reduces toxic effects by facilitating penetration into infective tissues/cells/ macrophages and hindering its clearance from the site of action. For example, Berberine-loaded liposomes for the treatment of L. infantum-infected BALB/c mice. The synthesized Berberine-loaded liposomes enhanced its selectivity index more than 7 -fold by decreasing its cytotoxicity to macrophages (Calvo et al., 2020). In an in vivo studies, Berberine-loaded liposomes enhanced the drug accumulation in liver and spleen of mice. Further, lipid nanoparticles [particularly solid-lipid nanoparticles (SLNs)] are composed of solid bio-degradable matrix with physiological lipids. SLNs have significant properties and gained some advantages like affordable cost, safety, smaller size and higher drug loading capacity. Shabi Parvez et al. prepared 2hydroxypropyl- $\beta$-cyclodextrin modified solid-lipid nanoparticles (m-DDSLNs) for oral delivery of Amphotericin $\mathrm{B}(\mathrm{AmB})$ and Paromomycin (PM) against murine visceral leishmaniasis. The in vitro anti-leishmanial activity of m-DDSLNs $(1 \mu \mathrm{g} / \mathrm{ml})$ showed maximum inhibition (96.22\%) of $L$. donovani amastigote formation. The liver parasite burden was also significantly decreased by m-DDSLNs $(20 \mathrm{mg} / \mathrm{kg} \times$ 5 days, p.o.) $(p<0.01)$ relative to miltefosine $(3 \mathrm{mg} / \mathrm{kg} \times$ 5 days, p.o.) in L. donovani-infected BALB/c mice. The observed results demonstrated that the site specific delivery of SLNs (Parvez et al., 2020).

Additionally, numerous techniques have been used to design nanoparticulate systems for targeted delivery of drugs competently to the infection site. In this way, the macrophages take up the drug-loaded nanocarrier by phagocytosis, where they will directly act on the parasites Figure 1 illustates the phagocytosis process of absorption of drug loaded nanoparticles. In one of these techniques, a ligand conjugated nanoparticles to recognize and bind to the cell surface receptors that are over expressed on the desired target (macrophage) has been used (Blanco et al., 2015). Leishmanial parasites are an intracellular parasite that mainly attacks macrophages, which can inhibit phagosomes maturation so as to survive and replicate within the macrophages. Macrophage-targeted drug delivery systems might overcome many of the associated problems such as drug toxicity, resistance, stability, etc., (Yasinzai et al., 2013). For example, in the management of VL, the production of 
rifampicin (RIF) encapsulated mannose-conjugated chitosan nanoparticulate system for selective delivery to macrophages has been reported (Jain et al., 2013). This mannose-conjugated chitosan nanoparticulate system loaded with rifampicin can recognize and bind the overexpressed mannose receptor on the surface of macrophages, and then nanocarrier (chitosan nanoparticles) can penetrate the cells within macrophages and deliver the loaded drugs efficiently. The efficacy of orally administered paromomycin (PM)-loaded mannosylated thiomeric nanoparticles (MTC-PLGA-PM) for targeted delivery to pathological organs against VL therapy has also been evaluated in an another report. The observed ex vivo permeation results showed that PM permeation with MTCPLGA-PM against free PM was 12.73 times higher. In the $L$. donovani contaminated macrophage model, flow cytometry analysis suggested the enhanced macrophage uptake and parasite killing behavior (Chaubey and Mishra, 2014).

\section{Nano-Engineered Particles for the Treatment of Chagas Disease}

Longer circulation time in body (long half-life time), therapeutic dose at the disseminated intracellular parasite infection site, high stability, highly effective against both chronic and acute phase of infections are essentially required for any drugs used in the treatment of Chagas disease. But available trypanocidal agents (Nifurtimox or Benznidazole) fails to reach the infection site of the amastigotes nests, due to nature of plasma membrane and the rigid microenvironment of the host cells (Sánchez et al., 2002). On the other side, non-targeted distribution of trypanocidal drugs produces toxic effects and develop drug resistance against pathogens. Several studies shows that nano-engineered carrier/vehicle modifies the physico-chemical properties of conventional drugs, like small size, prolonged circulation time, selective targetting to cells/tissues, cross the intracellular barriers and greatly delivered the trypanocidal drugs into infection site (Chowdhury et al., 2017). The selected nano-engineered materials should be bio-degradable with maximum loading capability of a variety of trypanocidal agents and enhanced delivery of loadeddrugs into target site in a sustainable manner. Size of the nanoparticles play a major role in the treatment of Chagas disease (Seremeta et al., 2019). For example, size less than $200 \mathrm{~nm}$ enables a longer blood stream circulation period and avoids accumulation in the liver and spleen. In addition, surface characteristics (charge) can also play a major role in protein adsorption, which in turn affects nanoparticles' pharmacokinetics and bio-distribution profiles (Kunjiappan et al., 2020). Research has shown that highly cationic nanocarrier systems are very easily removed from circulation compared to highly anionic nanocarrier systems. Compared to neutral nanocarrier and those with small negative charge, the circulating half-lives are considerably prolonged since the negative aspect of the cell membranes allows them to significantly absorbed by the cells. The nano-formulation of the trypanocidal drugs against various forms of $T$. cruzi parasites consider that the uptake process is only present in mammalian host cells and epimastigotes. In this view, polymeric nanoparticles, solid-lipid nanoparticles, liposomes, mesoporous silica nanoparticles, micelles, nanoemulsion are majorly used for trypanocidal drug delivery against Chagas disease (Blanco et al., 2015).

Polymeric nanoparticles can be loaded with trypanocidal drugs, and adjuvants in vaccines are dissolved or encapsulated/entrapped within or surface-adsorbed onto the polymeric core. Polymeric nanoparticles within the size range from 1 to $1,000 \mathrm{~nm}$ can be synthesized using natural (chitosan, gelatin, alginate, and albumen) and synthetic polymers (PEG, polycyanoacrylate, PLA, PCL, PLGA. These polymers are widely used, which are non-toxic, bio-degradable, permeable, FDA approved and bio-compatible. Gonzalez-Martin et al. (1998) developed poly (alkylcyanoacrylate) nanoparticles (PACA) for targeted delivery of nifurtimox against T. cruzi, accountable for Chagas' disease. Nifurtimox loaded PACA nanoparticles were formulated by an emulsion polymerization reaction of longer alkyl chains, formed polymer leads to slower degradation, less cytotoxic and sustainable drug release pattern. After $6 \mathrm{~h}$ of incubation at $\mathrm{pH} 7.4$, the prepared nanoparticles were less than $200 \mathrm{~nm}$ in size, with $33.4 \%$ absorption and $65.4 \%$ nifurtimox drug release rate. In vitro assessment using $\mathrm{T}$ cultures, compared to standard solution of nifurtimox, T. cruzi epimastigotes showed dramatically increased trypanocidal activity. Studies of cell cultures previously infected with metacyclic parasites found that only $2 \mathrm{~h}$ of $0.001 \%$ nanoparticle suspension decreased parasitism by $87-94 \%$, both when nanoparticles were filled with nifurtimox as well as unloaded. The bio-compatible polymeric nanoparticles composed of chitosan/sodium tripolyphosphate (TPP) were developed by Seabra and Durán (2017) and used to encapsulate thiol-containing mercaptosuccinic acid (MSA). Nitrosation with sodium nitrite $\left(\mathrm{NaNO}_{2}\right)$ was involved in the free thiol group of MSA, leading to the development of nanoparticles containing S-nitroso-MSA. With the release of free nitrogen, these polymeric nanoparticles serve as nitrogen donors. NO-releasing nanoparticles treated with cells displayed substantial decrease in the \% of infected macrophages (with amastigotes). Liposomes are vesicular nanomaterial structures formed by lipid bilayers of phospholipid and cholesterol. The use of natural phospholipids for liposome formulations possessing non-immunogenic, less toxic, higher stability, physiologically inert, tissue selectivity and delayed release of loaded active medicaments. These systems can deliver variety of molecules such as chemotherapeutics, siRNA, ribosomes, peptides, proteins, DNA, adjuvants in vaccines while providing significant therapeutic effects with minimal toxicity. For instance, Morilla et al. (2005) formed hydrogenated soybean phosphatidylcholine, distearoyl-phosphatidylglycerol with cholesterol, composed of benznidazole (BNZ) loaded multilamellar liposomes/vesicles (MLV). The MLV, BNZ, reduced its plasma protein association by $45 \%$ and became refractory to the blood's sinking effect, falling 4.5 times. In addition, $\mathrm{BNZ}$ had a greater volume of distribution $(160 \pm 20$ vs. $102 \pm 15 \mathrm{ml} / \mathrm{kg})$ and overall clearance $(35.23 \pm 2.3$ vs. $21.9 \pm 1.4 \mathrm{ml} / \mathrm{h} \mathrm{kg})$ when loaded into MLV, and a lower concentration time curve ( $7.23 \pm 0.2$ vs. $9.16 \pm$ $0.5 \mu \mathrm{g} \mathrm{h} / \mathrm{ml}$ ) than BNZ. The BNZ loaded MLV are more rapidly cleared from circulation than free benznidazole due to lower 
interaction with blood components and hence lower side effects can be expected. A mimetic lipid membranes based on lipids extracted from macrophages as a strategy to controlled release of the trypanocidal agent $N, N^{\prime}$-Squaramide 17 against $T$. cruzi has been reported (Quijia et al., 2020).

Solid-lipid nanoparticles (SLNs) composed of lipids have excellent physiological acceptability, bio-compatible, biodegradable with minimal human toxicity and can be used as novel pharmaceutical drug delivery systems. SLNs provides many benefits: they protect the drug against enzymatic degradation, controllable drug release, and dismiss the use of toxic organic solvents (Pandian et al., 2020). For the efficient treatment of Chagas disease, many researchers used SLNs, Carneiro et al. (2014), for example, developed 5-hydroxy-3-methyl-5-phenylpyrazoline-1-(S-benzyl dithiocarbazate) ( $\left.\mathrm{H}_{2} \mathrm{bdtc}\right)$-encapsulated SLNs against Chagas disease. Parasitemia in mice at clinically used concentration of $\mathrm{H}_{2}$ bdtc-loaded SLNs was 100 times lower than that usually used for BNZ (clinically applied at a concentration of $400 \mu \mathrm{mol} \mathrm{kg}{ }^{-1} \mathrm{day}^{-1}$ ). The $\mathrm{H}_{2}$ bdtc loaded SLNs also decreased liver, heart inflammation, lesions and resulted in $100 \%$ survival of $T$. cruzi infected mice.

\section{Nano-Engineered Particles for the Treatment of Soil-Transmitted Helminthiasis}

Infection with soil-transmitted helminthiasis (STHs) is a public health concern, especially in rural areas in the tropical countries (Savioli and Albonico, 2004). In the list of the world's NTDs, STH is also included. The STHs include the Ascaris lumbricoides roundworm, the Trichuris trichiura whipworm, the hookworms, Necator americanus, Ancylostoma duodenale, and Strongyloides stercoralis (Phuphisut et al., 2014). An approximately 22.1 million disability adjusted life years (DALYs) due to hookworm, around 10.5 million due to $A$. lumbricoides are the worldwide burden of disease caused by these intestinal nematodes. Around 6.4 million due to $T$. lumbricoides and Trichuris trichiura bringing a total of 39 million DALYs combined. The wider community has recognized the significance of remembering STHs that their burden of disease is as high as that of tuberculosis (34.7 million DALYs) or malaria (46.5 million DALYs).

The targeting whole group of people regardless of symptoms by mass drug administration is one method for managing the disease in places where it is widespread. For this reason, the drug of choice suggested by WHO are broad-spectrum benzimidazoles, such as MBZ and ABZ. These single-dose anthelmintics are safe, relatively inexpensive, and effective for several months. MBZ can be prescribed for three consecutive days with a single dose twice a day. A single dose of $A B Z$ is administered. Combination treatment with ivermectin and diethylcarbamazine is advocated in cases of co-infection. Clinical trials have shown that the novel drug tribendimidine, which was approved in China by CCDC for human use in the year 2004 is highly effective against major human flukes, ascaris ( $>90 \%$ cure rate) and hookworm (>82\%); however, with a low whipworm cure rate $(<37 \%)$ (Crompton, 2001).
MBZ was the first commonly used STH drug, but albendazole $\mathrm{ABZ}$ showed better anti-worm effect, so that the amount of metabolites produced in the serum from albendazole is up to 10 times higher than that of metabolites produced from MBZ. ABZ is an inexpensive drug, even at deficient concentration $(7.5 \mathrm{mg} / \mathrm{kg})$, shows anthelminthic activity. Even after several administrations, ABZ is surprisingly healthy at high doses (600 mg). However, ABZ has its drawbacks, including poor water solubility, poor gastrointestinal absorption (5\%), rapid protein synthesis, and dissemination to other non-target tissues. To maximize therapeutic effectiveness, several doses of $\mathrm{ABZ}$ also needed to be administered. Therefore, alternative novel drug delivery formulations of $\mathrm{ABZ}$ need to be produced to increase its intestinal absorption and decrease its side effects during routine treatment. Consequently, many attempts were made to address these issues. Several formulation methods such as particle size reduction, complexation, emulsion, and suspension, have been studied to enhance the solubility and bio-availability of this drug. In addition, it has also been discussed about the development of novel nano formulations (Muchiri et al., 2001).

Nanotechnology offers one method to solve the limitations of drugs and their traditional pharmaceutical formulations. Several studies have been reported in bio-medical nanoparticles (NP) for their utility in enhancing the bio-availability as well as in the treatment and prevention of several diseases. Ultimately, utilizing the carrier method, including liposomes, cyclodextrins and nanoparticles are the techniques to solve these issues. In recent years, several studies have centered on the development of novel ABZ-containing nano formulations such as albumin nanoparticles, solid-lipid nanoparticles, chitosantripolyphosphate nanoparticles, and chitosan-PLGA nanoparticles have been ynthesized and examined.

Cyclodextrins (CDs) and other polymers that have been commonly used with water-insoluble pharmaceutical drugs to improve their solubility and availability, among the several pharmaceutical techniques developed to increase drug efficacy. It prepares $\mathrm{ABZ}$ formulations, including $\beta$-cyclodextrin $(\beta C D)$ or hydroxypropyl- $\beta$-cyclodextrin (HP $\beta C D)$, which are or are not connected with the water-soluble polymer PVP. These formulations had high solubility, stability at dissolution rates and slightly increased anthelmintic effect (Pacheco et al., 2018).

To enhance solubility and cytotoxicity, chitosan and tripolyphosphate nanoparticles containing $\mathrm{ABZ}$ were prepared. In experimentally infected mice, delivery of $\mathrm{ABZ}$ as lipid nano capsules improved the oral bio-availability and effectiveness of cystic echinococcosis therapy. Similarly, for the treatment of Toxocara canis infection in vivo, ABZ loaded SLNs were examined. Furthermore, the nanoencapsulation of ABZ in PLGA nanoparticles coated with chitosan could boost its activity. SLNs have been used as an effective carrier device since early 1990s, among the numerous nanocarriers available. These nanoparticles have advantages compared to other colloidal carriers, such as regulating drug release, drug targeting, improving the chemical stability of drugs, functioning as a carrier for lipophilic and hydrophilic drugs as well as with maximum potential for mass production (Kang et al., 2017). 
Once thoroughly characterized in terms of structure, morphology, scale, and surface characteristics, magnetic nanoparticles (MNPs) are applied as carrier for the efficient delivery of ABZ. For the achievement of enhanced drug loading, controlled drug release, distribution, the best composition and tunable properties were optimized. Uniform magnetic solid-lipid NPs (MSLNPs) core/shell structure multifunctional mesoporous nano-capsules, consisting of inorganic $\left(\mathrm{Fe}_{3} \mathrm{O}_{4}\right)$ NPs as the core and stearic acid as the shell, have been described as potential platform for anti-parasitic drug delivery. Different techniques have identified and recognized these solid-lipid NPs (SLNPs) and MSLNPs possesses spherical particle morphology. Depending on ultrasound irradiation, reagent concentration and the order of addition, the average particle sizes of SLNPs and MSLNPs were between 20 and $120 \mathrm{~nm}$. The MSLNPs prepared using ultrasound were homogeneously distributed on the surface of SLNPs with a spherical form and little agglomeration. The in vitro release analysis showed that within $36 \mathrm{~h}, 84.0 \%$ of $\mathrm{ABZ}$ was released gradually. In addition, the synthesis method proposed was more rapid, eco-friendly compared to other strategies and has high efficiency for the delivery of ABZ (Abidi et al., 2018).

As new formulations are prepared, Chitosan nanoparticles are loaded with either ABZ or PZQ. Chitosan powder with various molecular weights were applied to the acetic acid solution containing Poloxamer 407 in order to synthesize nanoparticles. By adjusting one parameter, several parameters have been explored while others have been held constant to optimize the system. Particle size, polydispersity index, alpha potential, drug loading, and in vitro drug release have been assessed. The average sizes of the ABZ and PZQ loaded chitosan nanoparticles were 224.9 and $174.6 \mathrm{~nm}$, respectively. For ABZ, drug-loading and entrappment efficiencies were measured to be 0.55 and $11 \%$, respectively, while for PZQ, these were 0.53 and $22 \%$, respectively. The results showed that the size of the nanoparticles was determined by molecular weight of chitosan, chitosan concentration, chitosan-to-sodium tripolyphosphate ratio, and $\mathrm{pH}$ of solution. It seems that the current method is suitable for the production of PZQ-containing chitosan nanoparticles, but not sufficiently suitable for ABZ chitosan nanoparticles (Torabi et al., 2018).

Various formulations of $\mathrm{ABZ}$ and $\mathrm{MBZ}$ with an improved in vitro solubility profile have been developed $(\beta$-cyclodextrin inclusion complexes, chitosan-based microcrystals $(\mathrm{CH})$ and PVA and polysorbate 80 (P80) based nanoparticles and their activities have been tested in vitro and in vivo against the hookworm Heligmosomoides polygyrus. All tested formulations displayed faster, higher degree of dissolution and were more active than the regular products. ABZ-P80 demonstrated the highest improvement compared to $\mathrm{ABZ}$ in terms of increased solubility (4-fold increase) and in vivo activity (ED; $50 ; 7.0 \mathrm{mg} / \mathrm{kg}$ for $\mathrm{ABZ}$ and $4.1 \mathrm{mg} / \mathrm{kg}$ for $\mathrm{ABZ}-\mathrm{P} 80)$. Although, MBZ activity was lower than $A B Z$ in all situations, improved $\mathrm{MBZ}$ formulations were better than standard MBZ, where MBZ-CH demonstrated significantly higher in vivo activity (ED; 50; $8.02 \mathrm{mg} / \mathrm{kg}$ vs. ED; $50 ; 203 \mathrm{mg} / \mathrm{kg}$ for $\mathrm{MBZ}$ ). $\mathrm{MBZ}-\mathrm{CH}$ and ABZ-P80 formulations has been established as lead formulations in the reported work and further studies need to be performed (Buchter et al., 2020).

\section{Nano-Engineered Particles for the Treatment of African Trypanosomiasis/ Sleeping Sickness}

Human African Trypanosomiasis (HAT) or sleeping sickness is caused by trypanosome parasites mainly found in sub-Saharan Africa and transmitted by tsetse flies. Two Trypanosoma brucei sub-species cause illnesses: T. b. Gambians in Central and West Africa, and T. $b$. Rhodesia in Eastern Africa. It is one of the key causes of deaths in poor, disadvantaged regions of Africa, America and Asia (Prayag et al., 2020).

Today, the WHO suggests several treatment options for HAT, depending on the sub-species of parasites involved and the level of development of the disease, including pentamidine, suramin, melarsoprol, eflornithine, nifurtimox, and fexinidazole.

Pentamidine emerged in the year 1940 and has been used to treat the early phase of gHAT since then. It is an aromatic diamidine that has many unpleasant side effects, such as glucose homeostasis disorders, leukopenia, hypotension, as well as an inconvenient route of administration (intramuscular). In addition, it has poor permeability of the blood brain barrier (BBB), which suggests that it is not effective for late-stage HAT treatment. In the year 1920, Suramin entered the market and is still one of the treatment choices for the first step of rHAT. Urticarial rash is the most common side effect (which affects around $90 \%$ of the patients). Some adverse events include nephrotoxicity, pyrexia, and nausea that are reversible. An arsenic derivative, melarsoprol has been prescribed for the treatment of second stage of HAT for years. It has been used since 1949, but its toxicity is extremely high, causing, in some cases, serious encephalopathic syndrome along with high mortality rate. Eflornithine, a repurposed medication firstly investigated as an anticancer agent, is another approved drug for HAT treatment. As an alternative to melarsoprol, eflornithine has been used, and its mode of action includes inhibition of the ornithine decarboxylase enzyme. Its side effects are less serious than those of melarsoprol, but its poor oral bio-availability requires intravenous administration. When eflornithine is paired with nifurtimox, the result is greatly improved. As a monotherapy treatment in $T$. brucei. Nifurtimox is effective against both the early and late stages of Gambian infections, but after long-term administration it has very variable cure rate (30-80\%) and high toxicity. Fexinidazole has been approved for both hemolymphatic and meningoenchepalitic gHAT as the first oral drug, and is undergoing 5-years clinical trial to prove its effectiveness against rHAT. Fexinidazole is readily dispersed across the body, including the brain, following oral administration.

Oxaborole SCYX-7158 is currently undergoing clinical trials for HAT therapy. Encouraging SCYX-7158 outcomes in animal models has made the responsible researchers believe that late-stage HAT may be good treatment choice. In animal models of the disease, two other oxaborole compounds, SCYX1608210 and SCYX-1330682 also showed good efficiency. A 
placebo-controlled, randomized, double-blind study, evaluating the tolerability and pharmacokinetic parameters of SCYX-7158 was completed in the year 2015. The study indicated that the drug crosses the BBB quickly, thus being a successful candidate for late-stage HAT treatment, although some adverse effects have been observed, such as gastrointestinal reactions and headaches. Based on these findings, a phase II/III study to assess the efficacy and safety of SCYX-7158 as oral therapy for adult gHAT patients began in the year 2017. A single administration of $960 \mathrm{mg}$ was involved in the chosen dosage regimen. Results of Phase II/III are anticipated in the coming years.

Researchers and drug producers have long been ignored because of the lack of economic benefits from tropical parasitic infections. In order to minimize the deaths associated with this disease, there is an immense need for more effective, innovative strategies. Important efficacy advances have been seen in nanotechnological approaches to the delivery of current drugs with reduced doses.

As a promising field of research, nanoparticulate drug delivery systems of lipid, polymer or metal composition have emerged, as experimental evidence indicates that they can enhance the ability to directly target pathogens, penetrate barriers within the host allowing the drug to reach pathogen residence areas, minimize toxicity by reducing the amount and frequency of administration of the dose. In general, though retaining high specificity and selectivity, nanoparticulate systems can possess several synergistic functions. Scientists have been exploring co-delivery systems to boost bio-availability and effectiveness, as well as to treat different diseases with the same formulations, using the same delivery mechanism to encapsulate multiple drugs with synergistic properties. Through the combined use of nifurtimox and eflornithine, synergy is already utilized in the treatment of HAT and, as such, these multi-drug encapsulations may be especially effective against this parasite, although further studies are needed. There is also potential for these delivery mechanisms to specifically target the immune system, as well as the parasites themselves (Volpedo et al., 2019).

Restricted permeability by therapeutic agents across the bloodbrain barrier is one of the key limitations that an effective trypanocidal agent can present to treat late-stage HAT as a valid option. For example, once the parasite has entered the CNS, pentamidine is often ineffective, and such a lack of efficacy is thought to respond to its inability to reach the brain. The restricted bio-availability of pentamidine in the brain has been demonstrated in mice by Sanderson et al., whose findings indicated that active efflux mediated by P-glycoprotein and multidrug resistance-associated proteins conditioned the drug delivery to the brain. Different methods have been used for the delivery of drugs to the brain, such as intracerebroventricular administration or intranasal delivery. Among them, the permeability of polycaprolactone nanoparticles and liposomes containing both pentamidine across a monolayer of immortalized mouse brain endothelioma seems especially promising because of its flexibility and decreased side effects compared to other delivery options, including enhanced brain bio-availability via drug-carrier conjugates. The mean diameter of the pentamidine- loaded polycaprolactone nanoparticles was $267.6 \mathrm{~nm}$ and the zeta potential was $-28.1 \mathrm{mV}$, while the mean diameter of the liposomes was $119.6 \mathrm{~nm}$ and the zeta potential was $11.78 \mathrm{mV}$. Low dispersity and comparable loading capability was demonstrated by both the systems. After $24 \mathrm{~h}, 87$ and $66 \%$ of the doses were transported by liposomes and polycaprolactone nanoparticles, respectively. Besides, only $63 \%$ of the pentamidine dose was freely penetrated. The data indicated that lipid structures could be a promising nanocarriers to increase the pentamidine's brain bioavailability (Muraca et al., 2020).

In the treatment of Trypanosoma spp., SLNs has also shown promising results. Infections generally speaking, combined treatment with SLNs preparations has been shown to significantly boost curative outcomes relative to any medication used alone. For the treatment of Trypanosoma, combination therapy such as eflornithine-nifurtimox is currently commonly used. Infections with brucei and thus possible use of SLNs to amplify the healing properties of various medicines is of particular interest.

High pressure homogenization $(\mathrm{HPH})$ of the drug molecule and small amount of surfactant has been used as dispersant during preparation of nanosuspensions of drugs. Size is influenced by the consistency of the commodity and the number of cycles. The use of particular surfactants prevents RES detection and prolongs the length of blood circulation. For i.v., this technology is used. Poorly soluble drug melarsoprol nano suspension with poloxamer 188 or 407 and mannitol 72 was developed by Ben Zirar et al. The measurements were $324 \pm 88$ and $407 \pm 45 \mathrm{~nm}$, respectively. The nanosuspension size dependents on poloxamer 188 as well as increases depending on the concentration of melarsoprol. After a freeze-drying point, they were deposited, preventing the incidence of aggregation phenomena. The nanosuspension of melarsoprol must be administered immediately after reconstitution to restrict melarsoprol hydrolysis and the formation of melarsenoxide. The distribution to various tissues in mice demonstrated strong targeting of the reticuloendothelial system with 5-to-9-fold higher in the liver concentration than the free drug. The concentration in the brain was 3-5 times lower than that of the free drug. Their excessive scale, which makes it difficult to cross the BBB may explain these outcomes. The development of melarsoprol sustained release dosage (SRD) form is intended to avoid the injection of PEG and to improve its controlled release behavior. Microparticles of melarsoprol was prepared with PCL either by suspension-in-oil-in-water (S/O/W) solvent evaporation method $(34 \pm 17 \mu \mathrm{m})$ or by complexation of melarsoprol with methyl $\beta$-cyclodextrin followed by water-in-oilin-water $(\mathrm{WCD} / \mathrm{O} / \mathrm{W})$ solvent evaporation method $(31 \pm 17 \mu \mathrm{m})$. The surface of microparticles was observed by scanning electron microscopy: S/O/W emulsion showed rougher aspect with crystals on the polymeric surface and WCD/O/W emulsion showed smooth aspect with potential cavities. The S/O/W microparticles presented a melarsoprol incorporation of $161 \mathrm{mg} / \mathrm{g}$ and an in vitro $50 \%$ drug release after $2 \mathrm{~h}$ and $80 \%$ after $7 \mathrm{~h}$. Whereas, the microparticles $\mathrm{WCD} / \mathrm{O} / \mathrm{W}$ containing only $2.89 \mathrm{mg} / \mathrm{g}$ of melarsoprol presented a faster drug release which was incomplete in their in vitro conditions (phosphate buffer $\mathrm{pH} 7.4,30 \%$ PEG, $37^{\circ} \mathrm{C}$ ). The burst effect observed was 
TABLE 3 | Represents the list of nanoparticles under clinical trials.

\begin{tabular}{|c|c|c|c|c|}
\hline $\begin{array}{l}\text { S. } \\
\text { No }\end{array}$ & Molecules & Disease & Stage & References \\
\hline 1. & Chitosan & Leishmaniasis & $\begin{array}{l}\text { Pilot clinical } \\
\text { study }\end{array}$ & $\begin{array}{l}\text { Chitosan-based biocompatible dressing for treatment of recalcitrant lesions of } \\
\text { cutaneous leishmaniasis: A pilot clinical study. }\end{array}$ \\
\hline 2. & Liposomal amphotericin & $\begin{array}{l}\text { Disseminated } \\
\text { leishmaniasis }\end{array}$ & Phase 3 & NCT02025491 \\
\hline 3. & Liposomal amphotericin B & $\begin{array}{l}\text { Indian visceral } \\
\text { leishmaniasis }\end{array}$ & Phase 3 & NCT00628719 \\
\hline 4. & $\begin{array}{l}\text { Liposomal meglumine } \\
\text { antimoniate }\end{array}$ & $\begin{array}{l}\text { Cutaneous } \\
\text { leishmaniasis }\end{array}$ & Early phase 1 & NCT01050777 \\
\hline 5. & $\begin{array}{l}\text { Topical amphotericin-B } 0.4 \% \\
\text { liposomal gel }\end{array}$ & $\begin{array}{l}\text { Cutaneous } \\
\text { leishmaniasis }\end{array}$ & Phase 2 & NCT02656797 \\
\hline 6. & Leish-111f + MPL-SE vaccine & Mucosal leishmaniasis & Phase 1 & NCT02656797 \\
\hline
\end{tabular}

relied on the breakage of the aqueous cavities embedded in the polymeric structure. Due to high drug release observed, no further studies were performed on HAT infected animals. In addition, as melarsoprol is extremely toxic, it would be better to avoid its use even with drug delivery systems.

Nanoparticles of Pentamidine Methacrylate (PMAc) polymers have been well tolerated for several years as cement for implants in humans. Using a mixture of acrylic and methacrylic copolymers, PMAc NPs was prepared using emulsion polymerization. In PMAc NPs, pentamidine methane sulfonate was loaded via an ionic process involving free polymer carboxylic acid groups in order to treat VL. With decent tolerability, the efficacy was six times higher than free pentamidine. In vitro experiments suggested that PMAc NPs was absorbed by macrophages, which could minimize the interest of such NPs in the treatment of HAT. In addition, it was found that PMAc NPs has poor bio-degradability. Thus, pentamidine was developed as a polymer (D, L-lactide) with polyester polymer that is fully bio-degradable and bio-compatible. This polyester is slowly hydrolyzed into lactic acid, a metabolite of the cancer cycle, without enzyme action. This reaction is based on temperature and $\mathrm{pH}$. In the presence of Poloxamer 188 and lecithin, the PLA NPs was prepared by nano precipitation method. The phospholipid concentration limited by its solubility in acetone has affected the pentamidine base binding percentage. The monodispersed colloidal suspension was obtained with a mean diameter between 131 and $154 \mathrm{~nm}$ and is stable at $4^{\circ} \mathrm{C}$ for at least 9 months (Kroubi et al., 2011).

A surface coat consisting of single variant surface glycoprotein (VSG) covers the surface membrane of African tripanosomes that protects against lytic factors in human plasma and allows them to escape from the host immune reaction. This coat is re-cycled at very high velocity by endocytosis, rendering the surface of the trypanosome an ideal target for trypanocidal drug discovery and delivery. Covalently attached (PEGylated) to PLGA drug nanocarrier based on PEG to produce PEGylated PLGA nanoparticles. This nanocarrier was coupled to fragment of single domain heavy chain antibody (nanobody) that precisely recognizes the surface of pathogenic $T$. brucei protozoan. Pentamidine, the first-line drug for T. brucei was filled with nanoparticles. An in vitro efficacy assay showed a 7-fold decrease in the half-inhibitory concentration compared to the free drug in the formulation. In addition, in vivo therapy against
African trypanosomiasis murine model showed that the formulation has cured all infected mice at a dose 10 times lower than the minimum full curative dose of free pentamidine and $60 \%$ of mice at a dose 100 times lower. This nanocarrier was equipped with components approved for human use and filled with medicinal product currently in use for the treatment of the disease. In addition, this versatile nanobody-based device can be modified to load any drug, opening up a number of new potential therapies for other diseases as well. This study showed that due to high turnover of the entire cell membrane, the trypanosome surface is an excellent therapeutic target. The small size of nanobodies enable them to enter preserved epitopes that are inaccessible to traditional antibodies found in places. Nanobody conjugation was necessary for both in vitro and in vivo experiments in order to access the effectiveness of the formulations (Arias et al., 2015; Stijlemans et al., 2017).

Trypnocidal drug pentamidine-loaded functionalized PEGylated-chitosan nanoparticles, coated by a single-domain antibody (nanobody) derived from camel heavy-chain antibodies, which targets the surface of T. brucei. If loaded into this nanocarrier, instead of via classical cell surface transporters, pentamidine enters trypanosomes via endocytosis. In a murine model of acute African trypanosomiasis, the curative dose of pentamidine-loaded nanobody-chitosan nanoparticles was 100 fold lower than pentamidine alone. Crucially, due to mutations in the surface transporter aquaglyceroporin, this new formulation showed undiminished in vitro and in vivo activity against trypanosome cell line that developed resistant to pentamidine (Unciti-Broceta et al., 2015).

\section{Nanoparticles for the Treatment of Dengue}

Antiviral drugs have been shown in a number of trials to be a promising option in the future. Unfortunately, these compounds usually have poor bio-availability and are resistant to viruses, which is why many methods to address this restriction rely on the use of number of nanocarrier systems, such as inorganic nanoparticles, liposomes, stable lipid nanoparticles, nanoemulsions, and polymeric nanoparticles, among others. Bio-degradable nanoparticles are also currently used as vaccine adjuvants (Chowdhury et al., 2017). Polymeric nanoparticles with adsorbed or entrapped antigens represent an innovative mechanism for delivering loaded immunogens to antigenpresenting cells selectively (Khalid and El-Sawy, 2017). For example, mice immunized with BSA nanoparticles adsorbed 
with all four serotypes of inactivated dengue virus developed an anti-dengue virus IgG response. Although, the nanoparticle mechanism induces humoral responses against dengue fever under both pre and post-infection condition (Hunsawong et al., 2015). Paul et al. found that cationic gold nanoparticles with siRNA complexe could penetrate vero cells and substantially reduces dengue virus serotype 2 (DENV-2) replication and infectious virion release. Metal nanoparticles (copper, silver, gold, and selenium) have also been studied against dengue virus vector, such as larvicidal activity. The findings showed that metal nanoparticles have the ability to inactivate the mosquito vectors of dengue fever (genus Aedes).

\section{CONCLUSION AND FUTURE PERSPECTIVES}

There is a major challenge and huge investments are required to develop new drugs for the control and treatment of NTDs. Despite the rapid spread of parasitic diseases around the world, exciting treatment strategies are very much required to control these diseases. But available conventional drugs for the treatment of parasitic diseases are decade old, weak, expression of fatal toxic side effects and high resistance to disease-causing pathogens. Numerous studies were reported that re-formulation of conventional drugs via nanoformulations can significantly reduces the toxicity and increases the strength of therapeutic efficacy with low cost. Table 3 Represents the list of nanoparticles under clinical trials. Various nanoformulations (liposomes, solid-lipid nanoparticles, metal nanoparticles, polymeric nanoparticles) are effectively used to control several NTDs safely and reduces adverse toxic effects. According to the obtained results, the

\section{REFERENCES}

Abdelmaksoud, A., and Gupta, S. K. (2020). Management of Leprosy Patients in the Era of COVID-19. Dermatol. Ther. 33, e13631. doi:10.1111/dth.13631

Abidi, H., Ghaedi, M., Rafiei, A., Jelowdar, A., Salimi, A., Asfaram, A., et al. (2018). Magnetic Solid Lipid Nanoparticles Co-loaded with Albendazole as an Antiparasitic Drug: Sonochemical Preparation, Characterization, and In Vitro Drug Release. J. Mol. Liquids 268, 11-18. doi:10.1016/j.molliq.2018.06.116

Abulaihaiti, M., Wu, X.-W., Qiao, L., Lv, H.-L., Zhang, H.-W., Aduwayi, N., et al. (2015). Efficacy of Albendazole-Chitosan Microsphere-Based Treatment for Alveolar Echinococcosis in Mice. Plos Negl. Trop. Dis. 9, e0003950. doi:10.1371/ journal.pntd.0003950

Ahmed, K. M., Gupta, B., and Gupta, R. (2018). Leishmaniasis Research in India: A Scientometric Assessment of Publications during 2008-17. Int. J. Med. Public Health 8, 96-103.

Al-Kamel, M. A. (2017). Stigmata in Cutaneous Leishmaniasis: Historical and New Evidence-Based Concepts. Our Dermatol. Online 8, 81-90. doi:10.7241/ ourd.20171.21

Allahverdiyev, A., Abamor, E. Ş., Bagirova, M., Ustundag, C. B., Kaya, C., Kaya, F., et al. (2011). Antileishmanial Effect of Silver Nanoparticles and Their Enhanced Antiparasitic Activity under Ultraviolet Light. Ijn 6, 2705. doi:10.2147/ ijn.s23883

Allahverdiyev, A. M., Abamor, E. S., Bagirova, M., and Rafailovich, M. (2011). Antimicrobial Effects of TiO2and Ag2O Nanoparticles against Drug-Resistant nanoparticulate mediated drug delivery system enhances the efficacy via site-specific delivery, higher targeting efficiency and increased the bio-availability of drug at disease site. Apart from this, ligand-conjugated drug-loaded-nanoparticles (for example, mannose-conjugated chitosan nanoparticles) have shown promising results for controlling NTDs. Ligandconjugated nanoparticles can be recognized, and bonded on the surface of macrophages and initiated to deliver the loaded drugs. Nanotechnology-enabled vaccines are a new strategy to successfully eliminate NTDs; at present, there are no vaccines against NTDs, but studies are undertaking to find effectual nano vaccines. In this review, several reported results indicated that re-formulation of conventional drugs via nanoparticles can improve the quality, efficacy and reduces the toxicity of drugs. However, nano-based vaccines are required to advance studies and research to develop effective, safe drugs with low cost against NTDs.

\section{AUTHOR CONTRIBUTIONS}

SK, SR and MS (8th author) conceived and designed the research. SR, SK, TP, PP, SG, VR, PP, MS (7th author) and MS (8th author) analyzed the data. SK, SR and MS (8th author) wrote the manuscript. All authors read and approved the manuscript.

\section{ACKNOWLEDGMENTS}

The authors are grateful to the Management of Kalasalingam Academy of Research and Education, Krishnankoil, India, for research fellowships and utilizing research facilities.

Bacteria Andleishmaniaparasites. Future Microbiol. 6, 933-940. doi:10.2217/ fmb.11.78

Alnasser, Y., Ferradas, C., Clark, T., Calderon, M., Gurbillon, A., Gamboa, D., et al. (2016). Colorimetric Detection of Plasmodium Vivax in Urine Using MSP10 Oligonucleotides and Gold Nanoparticles. PLoS Negl. Trop. Dis. 10, e0005029. doi:10.1371/journal.pntd.0005029

Alquadeib, B. (2016). Pharmaceutical Studies of Amphotericin-B: Oral Formulation Using Nanotechnology. Newcastle upon Tyne, United Kingdom: Newcastle University.

Amato, V. S., Tuon, F. F., Bacha, H. A., Neto, V. A., and Nicodemo, A. C. (2008). Mucosal Leishmaniasis. Acta tropica 105, 1-9. doi:10.1016/j.actatropica.2007.08.003

Andrews, P., Thomas, H., Pohlke, R., and Seubert, J. (1983). Praziquantel. Med. Res. Rev. 3, 147-200. doi:10.1002/med.2610030204

Apt, W. (2010). Current and Developing Therapeutic Agents in the Treatment of Chagas Disease. Dddt 4, 243-253. doi:10.2147/dddt.s8338

Arce-Fonseca, M., Rios-Castro, M., Carrillo-Sánchez, Sdel. C., Martínez-Cruz, M., and Rodríguez-Morales, O. (2015). Prophylactic and Therapeutic DNA Vaccines against Chagas Disease. Parasit Vectors 8, 121-127. doi:10.1186/ s13071-015-0738-0

Arias, J. L., Unciti-Broceta, J. D., Maceira, J., Del Castillo, T., Hernández-Quero, J., Magez, S., et al. (2015). Nanobody Conjugated PLGA Nanoparticles for Active Targeting of African Trypanosomiasis. J. Controlled Release 197, 190-198. doi:10.1016/j.jconrel.2014.11.002

Bambole, V., and Yakhmi, J. V. (2016). Tissue Engineering: Use of Electrospinning Technique for Recreating Physiological Functions. Nanobiomaterials in Soft 
Tissue Engineering, 5. Elsevier, 387-455. doi:10.1016/b978-0-323-428651.00014-3

Bank, W. (2017). Drug-resistant Infections: A Threat to Our Economic Future. Washington, DC, USA: World Bank.

Barrett, M. P., Boykin, D. W., Brun, R., and Tidwell, R. R. (2007). Human African Trypanosomiasis: Pharmacological Re-engagement with a Neglected Disease. Br. J. Pharmacol. 152, 1155-1171. doi:10.1038/sj.bjp.0707354

Bartelds, R., Nematollahi, M. H., Pols, T., Stuart, M. C. A., Pardakhty, A., Asadikaram, G., et al. (2018). Niosomes, an Alternative for Liposomal Delivery. PLoS One 13, e0194179. doi:10.1371/journal.pone.0194179

Becker, S. L., Liwanag, H. J., Snyder, J. S., Akogun, O., Belizario., V., Jr, Freeman, M. C., et al. (2018). Toward the 2020 Goal of Soil-Transmitted Helminthiasis Control and Elimination. PLoS Negl. Trop. Dis. 12, e0006606. doi:10.1371/ journal.pntd.0006606

Beltrán-Gracia, E., López-Camacho, A., Higuera-Ciapara, I., Velázquez-Fernández, J. B., and Vallejo-Cardona, A. A. (2019). Nanomedicine Review: Clinical Developments in Liposomal Applications. Cancer Nanotechnology 10, 1-40. doi:10.1186/s12645-019-0055-y

Berenguer, D., Alcover, M., Sessa, M., Halbaut, L., Guillén, C., Boix-Montañés, A., et al. (2020). Topical Amphotericin B Semisolid Dosage Form for Cutaneous Leishmaniasis: Physicochemical Characterization, Ex Vivo Skin Permeation and Biological Activity. Pharmaceutics 12, 149. doi:10.3390/pharmaceutics12020149

Biffi, S., Voltan, R., Bortot, B., Zauli, G., and Secchiero, P. (2019). Actively Targeted Nanocarriers for Drug Delivery to Cancer Cells. Expert Opin. Drug Deliv. 16, 481-496. doi:10.1080/17425247.2019.1604679

Blanco, E., Shen, H., and Ferrari, M. (2015). Principles of Nanoparticle Design for Overcoming Biological Barriers to Drug Delivery. Nat. Biotechnol. 33, 941-951. doi:10.1038/nbt.3330

Bonizzoni, M., Gasperi, G., Chen, X., and James, A. A. (2013). The Invasive Mosquito Species Aedes albopictus: Current Knowledge and Future Perspectives. Trends Parasitology 29, 460-468. doi:10.1016/ j.pt.2013.07.003

Brun, R., Blum, J., Chappuis, F., and Burri, C. (2010). Human African Trypanosomiasis. The Lancet 375, 148-159. doi:10.1016/s0140-6736(09)60829-1

Buchter, V., Priotti, J., Leonardi, D., Lamas, M. C., and Keiser, J. (2020). Preparation, Physicochemical Characterization and In Vitro and In Vivo Activity against Heligmosomoides Polygyrus of Novel Oral Formulations of Albendazole and Mebendazole. J. Pharm. Sci. 109, 1819-1826. doi:10.1016/ j.xphs.2020.02.002

Bustamante, D. M., De Urioste-Stone, S. M., Juárez, J. G., and Pennington, P. M. (2014). Ecological, Social and Biological Risk Factors for Continued Trypanosoma Cruzi Transmission by Triatoma dimidiata in Guatemala. PLoS One 9, e104599. doi:10.1371/journal.pone.0104599

Cabral, F. V., Pelegrino, M. T., Sauter, I. P., Seabra, A. B., Cortez, M., and Ribeiro, M. S. (2019). Nitric Oxide-Loaded Chitosan Nanoparticles as an Innovative Antileishmanial Platform. Nitric Oxide 93, 25-33. doi:10.1016/ j.niox.2019.09.007

Calvo, A., Moreno, E., Larrea, E., Sanmartín, C., Irache, J. M., and Espuelas, S. (2020). Berberine-Loaded Liposomes for the Treatment of Leishmania Infantum-Infected BALB/c Mice. Pharmaceutics 12, 858. doi:10.3390/ pharmaceutics 12090858

Capela, R., Moreira, R., and Lopes, F. (2019). An Overview of Drug Resistance in Protozoal Diseases. Int. J. Mol. Sci. 20, 5748. doi:10.3390/ijms20225748

Carneiro, Z. A., Maia, P. I. D. S., Sesti-Costa, R., Lopes, C. D., Pereira, T. A., Milanezi, C. M., et al. (2014). In Vitro and In Vivo Trypanocidal Activity of $\mathrm{H}$ 2 Bdtc-Loaded Solid Lipid Nanoparticles. Plos Negl. Trop. Dis. 8, e2847. doi:10.1371/journal.pntd.0002847

Chatelain, E., and Ioset, J.-R. (2011). Drug Discovery and Development for Neglected Diseases: the DNDi Model. Drug Des. Dev. Ther. 5, 175-181. doi:10.2147/DDDT.S16381

Chaubey, P., and Mishra, B. (2014). Mannose-conjugated Chitosan Nanoparticles Loaded with Rifampicin for the Treatment of Visceral Leishmaniasis. Carbohydr. Polym. 101, 1101-1108. doi:10.1016/j.carbpol.2013.10.044

Chowdhury, A., Kunjiappan, S., Panneerselvam, T., Somasundaram, B., and Bhattacharjee, C. (2017). Nanotechnology and Nanocarrier-Based Approaches on Treatment of Degenerative Diseases. Int. nano Lett. 7, 91-122. doi:10.1007/s40089-017-0208-0
Chowdhury, F. I., Kabir, A., Das, A., Mukerrama, S. M., and Masud, S. (2012). Chikungunya Fever: an Emerging Threat to Bangladesh. J. Med. 13, 60-64.

Conterno, L. O., Turchi, M. D., Corrêa, I., and De Barros Almeida, R. a. M. (2020). Anthelmintic Drugs for Treating Ascariasis. Cochrane Database Syst. Rev. 4, 1-101. doi:10.1002/14651858.CD010599.pub2

Crompton, D. (2001). Ascaris and Ascariasis. Adv. Parasitol. 48, 285-375. doi:10.1016/s0065-308x(01)48008-0

Darlan, D. M., Tala, Z. Z., Amanta, C., Warli, S. M., and Arrasyid, N. K. (2017). Correlation between Soil Transmitted Helminth Infection and Eosinophil Levels Among Primary School Children in Medan. Open access Macedonian J. Med. Sci. 5, 142-146. doi:10.3889/oamjms.2017.014

Darvishi, M. M., Moazeni, M., Alizadeh, M., Abedi, M., and Tamaddon, A.-M. (2020). Evaluation of the Efficacy of Albendazole Sulfoxide (ABZ-SO)-loaded Chitosan-PLGA Nanoparticles in the Treatment of Cystic Echinococcosis in Laboratory Mice. Parasitol. Res. 119, 4233-4241. doi:10.1007/s00436-02006901-2

Das, S., Roy, P., Mondal, S., Bera, T., and Mukherjee, A. (2013). One Pot Synthesis of Gold Nanoparticles and Application in Chemotherapy of Wild and Resistant Type Visceral Leishmaniasis. Colloids Surf. B: Biointerfaces 107, 27-34. doi:10.1016/j.colsurfb.2013.01.061

Date, A. A., Joshi, M. D., and Patravale, V. B. (2007). Parasitic Diseases: Liposomes and Polymeric Nanoparticles versus Lipid Nanoparticles. Adv. Drug Deliv. Rev. 59, 505-521. doi:10.1016/j.addr.2007.04.009

De Figueiredo Diniz, L., Urbina, J. A., De Andrade, I. M., Mazzeti, A. L., Martins, T. a. F., Caldas, I. S., et al. (2013). Benznidazole and Posaconazole in Experimental Chagas Disease: Positive Interaction in Concomitant and Sequential Treatments. Plos Negl. Trop. Dis. 7, e2367. doi:10.1371/journal.pntd.0002367

De Silva, N. R., Brooker, S., Hotez, P. J., Montresor, A., Engels, D., and Savioli, L. (2003). Soil-transmitted Helminth Infections: Updating the Global Picture. Trends Parasitology 19, 547-551. doi:10.1016/j.pt.2003.10.002

Dias, J. C. P., Silveira, A. C., and Schofield, C. J. (2002). The Impact of Chagas Disease Control in Latin America: a Review. Memórias do Instituto Oswaldo Cruz 97, 603-612. doi:10.1590/s0074-02762002000500002

Duan, Y., Dhar, A., Patel, C., Khimani, M., Neogi, S., Sharma, P., et al. (2020). A Brief Review on Solid Lipid Nanoparticles: Part and Parcel of Contemporary Drug Delivery Systems. RSC Adv. 10, 26777-26791. doi:10.1039/d0ra03491f

Ebikeme, C. E. (2007). Amino Acid Transporters \& Amino Acid Metabolism in Trypanosoma Brucei Brucei. Glasgow, Scotland, United Kingdom: University of Glasgow. Available at: https://eleanor.lib.gla.ac.uk/record=b2612424.

Ehrenberg, N., Ehrenberg, J. P., Fontes, G., Gyapong, M., Rocha, E. M., Steinmann, P., et al. (2021). Neglected Tropical Diseases as a Barometer for Progress in Health Systems in Times of COVID-19. BMJ Glob. Health 6, e004709. doi:10.1136/bmjgh-2020-004709

Else, K. J., Keiser, J., Holland, C. V., Grencis, R. K., Sattelle, D. B., Fujiwara, R. T., et al. (2020). Whipworm and Roundworm Infections. Nat. Rev. Dis. Primers 6, 1-23. doi:10.1038/s41572-020-0171-3

Ferreira, J. G., Gava, S. G., Oliveira, E. S., Batista, I. C., Fernandes, G. D. R., Mourão, M. M., et al. (2020). Gene Expression Signatures in AML-12 Hepatocyte Cells upon Dengue Virus Infection and Acetaminophen Treatment. Viruses 12, 1284. doi:10.3390/v12111284

Ferreira, T. P., Haddi, K., Corrêa, R. F., Zapata, V. L., Piau, T. B., Souza, L. F., et al. (2019). Prolonged Mosquitocidal Activity of Siparuna Guianensis Essential Oil Encapsulated in Chitosan Nanoparticles. PLoS Negl. Trop. Dis. 13, e0007624. doi:10.1371/journal.pntd.0007624

Finlay, C. M., Walsh, K. P., and Mills, K. H. (2014). Induction of Regulatory Cells by Helminth Parasites: Exploitation for the Treatment of Inflammatory Diseases. Immunological Rev. 259, 206-230. doi:10.1111/imr.12164

Forsyth, W. O. (2020). Manipulation of Host Signalling for the Characterisation and Control of Dengue Fever. Avialable at: http://hdl.handle.net/11343/243013.

Frechet, J. M., and Hawker, C. J. (1989). "Synthesis and Properties of Dendrimers and Hyperbranched Polymers," in Comprehensive Polymer Science. Editors S. L. Aggarwal and S. Russo. 2nd Supp. (Oxford: Elsevier Science), 71-132. doi:10.1016/b978-0-08-096701-1.00242-1

Garchitorena, A., Sokolow, S., Roche, B., Ngonghala, C., Jocque, M., Lund, A., et al. (2017). Disease Ecology, Health and the Environment: a Framework to Account for Ecological and Socio-Economic Drivers in the Control of Neglected 
Tropical Diseases. Philos. Trans. R. Soc. B: Biol. Sci. 372, 20160128. doi:10.1098/ rstb.2016.0128

García, C. C., Quintana, V. M., Castilla, V., and Damonte, E. B. (2018). Towards Host-Cell Targeting Therapies to Treat Dengue Virus Infections. Front. AntiInfective Drug Discov. 7, 45-87. doi:10.2174/9781681085623118070004

Garg, R., and Dube, A. (2006). Animal Models for Vaccine Studies for Visceral Leishmaniasis. Indian J. Med. Res. 123, 439-454.

Gascon, J., Bern, C., and Pinazo, M.-J. (2010). Chagas Disease in Spain, the United States and Other Non-endemic Countries. Acta tropica 115, 22-27. doi:10.1016/j.actatropica.2009.07.019

Gherbawy, Y. A., Shalaby, I. M., El-Sadek, M. S. A., Elhariry, H. M., and Banaja, A. A. (2013). The Anti-fasciolasis Properties of Silver Nanoparticles Produced by Trichoderma harzianum and Their Improvement of the Anti-fasciolasis Drug Triclabendazole. Int. J. Mol. Sci. 14, 21887-21898. doi:10.3390/ijms141121887

Gohla, S., and Dingler, A. (2001). Scaling up Feasibility of the Production of Solid Lipid Nanoparticles (SLNs). Die Pharmazie 56, 61-63.

Gonzalez-Martin, G., Merino, I., Rodriguez-Cabezas, M. N., Torres, M., Nuñez, R., and Osuna, A. (1998). Pharmaceutics: Characterization and Trypanocidal Activity of Nifurtimox-containing and Empty Nanoparticles of Polyethylcyanoacrylates. J. Pharm. Pharmacol. 50, 29-35. doi:10.1111/jphp.1998.50.issue-5

Gottschall, D., Theodorides, V., and Wang, R. (1990). The Metabolism of Benzimidazole Anthelmintics. Parasitol. Today 6, 115-124. doi:10.1016/ 0169-4758(90)90228-v

Gour, J. K., Srivastava, A., Kumar, V., Bajpai, S., Kumar, H., Mishra, M., et al. (2009). Nanomedicine and Leishmaniasis: Future Prospects. FASEB J. 4, 495-499. doi:10.1096/fj.04-2747rev

Guery, R., Henry, B., Martin-Blondel, G., Rouzaud, C., Cordoliani, F., Harms, G., et al. (2017). Liposomal Amphotericin B in Travelers with Cutaneous and Muco-Cutaneous Leishmaniasis: Not a Panacea. PLoS Negl. Trop. Dis. 11, e0006094. doi:10.1371/journal.pntd.0006094

Gupta, P., Garcia, E., Sarkar, A., Kapoor, S., Rafiq, K., Chand, H. S., et al. (2019). Nanoparticle Based Treatment for Cardiovascular Diseases. Cardiovasc. Haematological Disorders-Drug Targets (Formerly Curr. Drug TargetsCardiovascular Hematological Disorders) 19, 33-44. doi:10.2174/ 1871529x18666180508113253

Halder, A., Das, S., Bera, T., and Mukherjee, A. (2017). Rapid Synthesis for Monodispersed Gold Nanoparticles in Kaempferol and Anti-leishmanial Efficacy against Wild and Drug Resistant Strains. Rsc Adv. 7, 14159-14167. doi:10.1039/c6ra28632a

Han, H. J., Ekweremadu, C., and Patel, N. (2019). Advanced Drug Delivery System with Nanomaterials for Personalised Medicine to Treat Breast Cancer. J. Drug Deliv. Sci. Technol. 52, 1051-1060. doi:10.1016/j.jddst.2019.05.024

Hoekendijk, D. J., Hill, P. C., and Sowerby, S. J. (2016). Rationale for Quality Assurance in Fecal Egg Monitoring of Soil-Transmitted Helminthiasis. Am. J. Trop. Med. Hyg. 95, 502-504. doi:10.4269/ajtmh.15-0463

Holden-Dye, L., and Walker, R. (2014). Anthelmintic Drugs and Nematocides: Studies in Caenorhabditis elegans. The C. elegans Research Community, WormBook, 1-29. doi:10.1895/wormbook.1.143.2

Hong, S.-T. (2018). Albendazole and Praziquantel: Review and Safety Monitoring in Korea. Infect. Chemother. 50, 1-10. doi:10.3947/ic.2018.50.1.1

Horton, J. (2017). Thiabendazole and Flubendazole. Kucers' the Use of Antibiotics. London, United Kingdom: CRC Press, 3338-3346.

Hossen, S., Hossain, M. K., Basher, M., Mia, M., Rahman, M., and Uddin, M. J. (2019). Smart Nanocarrier-Based Drug Delivery Systems for Cancer Therapy and Toxicity Studies: A Review. J. Adv. Res. 15, 1-18. doi:10.1016/ j.jare.2018.06.005

Hotez, P. J., Bundy, D. A., Beegle, K., Brooker, S., Drake, L., De Silva, N., et al. (2006). Helminth Infections: Soil-Transmitted Helminth Infections and Schistosomiasis. Disease Control Priorities in Developing Countries (New York: Oxford University Press). 2nd edition.

Hunsawong, T., Sunintaboon, P., Warit, S., Thaisomboonsuk, B., Jarman, R. G., Yoon, I.-K., et al. (2015). A Novel Dengue Virus Serotype-2 Nanovaccine Induces Robust Humoral and Cell-Mediated Immunity in Mice. Vaccine 33, 1702-1710. doi:10.1016/j.vaccine.2015.02.016

Jackson, Y., Wyssa, B., and Chappuis, F. (2020). Tolerance to Nifurtimox and Benznidazole in Adult Patients with Chronic Chagas' Disease. J. Antimicrob. Chemother. 75, 690-696. doi:10.1093/jac/dkz473
Jain, N. K., Mishra, V., and Mehra, N. K. (2013). Targeted Drug Delivery to Macrophages. Expert Opin. Drug Deliv. 10, 353-367. doi:10.1517/ 17425247.2013.751370

Jansen, A. M., Xavier, S. C., and Roque, A. L. R. (2015). The Multiple and Complex and Changeable Scenarios of the Trypanosoma Cruzi Transmission Cycle in the Sylvatic Environment. Acta tropica 151, 1-15. doi:10.1016/j.actatropica.2015.07.018

Jumaa, M., and Müller, B. W. (2000). Lipid Emulsions as a Novel System to Reduce the Hemolytic Activity of Lytic Agents: Mechanism of the Protective Effect. Eur. J. Pharm. Sci. 9, 285-290. doi:10.1016/s0928-0987(99)00071-8

Junior, P. a. S., Molina, I., Murta, S. M. F., Sánchez-Montalvá, A., Salvador, F., Corrêa-Oliveira, R., et al. (2017). Experimental and Clinical Treatment of Chagas Disease: a Review. Am. J. Trop. Med. Hyg. 97, 1289-1303. doi:10.4269/ ajtmh.16-0761

Kamaly, N., Yameen, B., Wu, J., and Farokhzad, O. C. (2016). Degradable Controlled-Release Polymers and Polymeric Nanoparticles: Mechanisms of Controlling Drug Release. Chem. Rev. 116, 2602-2663. doi:10.1021/ acs.chemrev.5b00346

Kang, B.-S., Choi, J.-S., Lee, S.-E., Lee, J.-K., Kim, T.-H., Jang, W. S., et al. (2017). Enhancing the In Vitro Anticancer Activity of Albendazole Incorporated into Chitosan-Coated PLGA Nanoparticles. Carbohydr. Polym. 159, 39-47. doi:10.1016/j.carbpol.2016.12.009

Kassebaum, N. J., Arora, M., Barber, R. M., Bhutta, Z. A., Brown, J., Carter, A., et al. (2016). Global, Regional, and National Disability-Adjusted Life-Years (DALYs) for 315 Diseases and Injuries and Healthy Life Expectancy (HALE), 1990-2015: a Systematic Analysis for the Global Burden of Disease Study 2015. The Lancet 388, 1603-1658. doi:10.1016/S0140-6736(16)31460-X

Katz, M., Despommier, D. D., and Gwadz, R. W. (1989). Parasitic Diseases. New York, NY: Springer, 208-259. doi:10.1007/978-1-4684-0327-5_5The Arthropods

Kealey, A. (2010). Neglected Tropical Diseases: Infection, Modeling, and Control. J. Health Care Poor Underserved 21, 53-69. doi:10.1353/hpu.0.0270

Kennedy, P. G. (2013). Clinical Features, Diagnosis, and Treatment of Human African Trypanosomiasis (Sleeping Sickness). Lancet Neurol. 12, 186-194. doi:10.1016/s1474-4422(12)70296-x

Khalid, M., and El-Sawy, H. S. (2017). Polymeric Nanoparticles: Promising Platform for Drug Delivery. Int. J. pharmaceutics 528, 675-691. doi:10.1016/ j.ijpharm.2017.06.052

Kroubi, M., Karembe, H., and Betbeder, D. (2011). Drug Delivery Systems in the Treatment of African Trypanosomiasis Infections. Expert Opin. Drug Deliv. 8, 735-747. doi:10.1517/17425247.2011.574122

Kulshrestha, A., Dhanawat, M., Das, N., and K Shrivastava, S. (2012). Small Molecules Antileishmanials: A Review. Lett. Drug Des. Discov. 9, 535-548. doi:10.2174/157018012800389359

Kumar, K., and Rai, A. (2011). Development and Evaluation of ProniosomeEncapsulated Curcumin for Transdermal Administration. Trop. J. Pharm. Res. 10, 697-703. doi:10.4314/tjpr.v10i6.1

Kunjiappan, S., Pavadai, P., Vellaichamy, S., Ram Kumar Pandian, S., Ravishankar, V., Palanisamy, P., et al. (2020). Surface Receptor-mediated Targeted Drug Delivery Systems for Enhanced Cancer Treatment: A State-of-the-art Review. Drug Develop. Res. 82 (3), 309-340. doi:10.1002/ddr.21758

Levine, R. (2007). Case Studies in Global Health: Millions Saved. Burlington, MA, USA: Jones \& Bartlett Publishers.

Li, M., Du, C., Guo, N., Teng, Y., Meng, X., Sun, H., et al. (2019). Composition Design and Medical Application of Liposomes. Eur. J. Med. Chem. 164, 640-653. doi:10.1016/j.ejmech.2019.01.007

Lidani, K. C. F., Andrade, F. A., Bavia, L., Damasceno, F. S., Beltrame, M. H., Messias-Reason, I. J., et al. (2019). Chagas Disease: from Discovery to a Worldwide Health Problem. Front. Public Health 7, 166. doi:10.3389/ fpubh.2019.00166

Lightowlers, M., Colebrook, A., Gauci, C., Gauci, S., Kyngdon, C., Monkhouse, J., et al. (2003). Vaccination against Cestode Parasites: Anti-helminth Vaccines that Work and Why. Vet. Parasitol. 115, 83-123. doi:10.1016/s0304-4017(03) 00202-4

Linares, E. M., Pannuti, C. S., Kubota, L. T., and Thalhammer, S. (2013). Immunospot Assay Based on Fluorescent Nanoparticles for Dengue Fever Detection. Biosens. Bioelectron. 41, 180-185. doi:10.1016/j.bios.2012.08.005

Lindner, A. K., Lejon, V., Chappuis, F., Seixas, J., Kazumba, L., Barrett, M. P., et al. (2020). New WHO Guidelines for Treatment of Gambiense Human African 
Trypanosomiasis Including Fexinidazole: Substantial Changes for Clinical Practice. Lancet Infect. Dis. 20, e38-e46. doi:10.1016/s1473-3099(19)30612-7

Lindoso, J. a. L., and Lindoso, A. a. B. (2009). Neglected Tropical Diseases in Brazil, Revista do Instituto de Medicina Trop. de São Paulo 51, 247-253. doi:10.1590/ s0036-46652009000500003

Long, N. V., Yang, Y., Teranishi, T., Thi, C. M., Cao, Y., and Nogami, M. (2015). Biomedical Applications of Advanced Multifunctional Magnetic Nanoparticles. J. nanoscience nanotechnology 15, 10091-10107. doi:10.1166/jnn.2015.11691

Longmire, M., Choyke, P. L., and Kobayashi, H. (2008). Clearance Properties of Nano-Sized Particles and Molecules as Imaging Agents. Considerations and caveats 3, 703-712. doi:10.2217/17435889.3.5.703

Look, M., Bandyopadhyay, A., Blum, J. S., and Fahmy, T. M. (2010). Application of Nanotechnologies for Improved Immune Response against Infectious Diseases in the Developing World. Adv. Drug Deliv. Rev. 62, 378-393. doi:10.1016/ j.addr.2009.11.011

Lustigman, S., Prichard, R. K., Gazzinelli, A., Grant, W. N., Boatin, B. A., Mccarthy, J. S., et al. (2012). A Research Agenda for Helminth Diseases of Humans: the Problem of Helminthiases. Plos Negl. Trop. Dis. 6, e1582. doi:10.1371/ journal.pntd.0001582

Mackey, T. K., Liang, B. A., Cuomo, R., Hafen, R., Brouwer, K. C., and Lee, D. E. (2014). Emerging and Reemerging Neglected Tropical Diseases: a Review of Key Characteristics, Risk Factors, and the Policy and Innovation Environment. Clin. Microbiol. Rev. 27, 949-979. doi:10.1128/cmr.00045-14

Madaan, K., Kumar, S., Poonia, N., Lather, V., and Pandita, D. (2014). Dendrimers in Drug Delivery and Targeting: Drug-Dendrimer Interactions and Toxicity Issues. J. Pharm. bioallied Sci. 6, 139-150. doi:10.4103/0975-7406.130965

Magalhaes, T., Robison, A., Young, M. C., Black, W. C., Foy, B. D., Ebel, G. D., et al. (2018). Sequential Infection of Aedes aegypti Mosquitoes with Chikungunya Virus and Zika Virus Enhances Early Zika Virus Transmission. Insects 9, 177. doi:10.3390/insects9040177

Malvy, D., and Chappuis, F. (2011). Sleeping Sickness. Clin. Microbiol. Infect. 17, 986-995. doi:10.1111/j.1469-0691.2011.03536.x

Martins-Melo, F. R., Carneiro, M., Ramos, A. N., Jr, Heukelbach, J., Ribeiro, A. L. P., and Werneck, G. L. (2018). The burden of Neglected Tropical Diseases in Brazil, 1990-2016: a Subnational Analysis from the Global Burden of Disease Study 2016. PLoS Negl. Trop. Dis. 12, e0006559. doi:10.1371/ journal.pntd.0006559

Mascarini-Serra, L. (2011). Prevention of Soil-Transmitted Helminth Infection. J. Glob. Infect. Dis. 3, 175-182. doi:10.4103/0974-777x.81696

Matsumura, Y., and Maeda, H. (1986). A New Concept for Macromolecular Therapeutics in Cancer Chemotherapy: Mechanism of Tumoritropic Accumulation of Proteins and the Antitumor Agent Smancs. Cancer Res. 46, 6387-6392.

Mazur, K. L., Feuser, P. E., Valério, A., Cordeiro, A. P., De Oliveira, C. I., Assolini, J. P., et al. (2019). Diethyldithiocarbamate Loaded in Beeswax-Copaiba Oil Nanoparticles Obtained by Solventless Double Emulsion Technique Promote Promastigote Death In Vitro. Colloids Surf. B: Biointerfaces 176, 507-512. doi:10.1016/j.colsurfb.2018.12.048

Mehrizi, T. Z., Khamesipour, A., Ardestani, M. S., Shahmabadi, H. E., Hoseini, M. H. M., Mosaffa, N., et al. (2019). Comparative Analysis between Four Model Nanoformulations of Amphotericin B-Chitosan, Amphotericin B-Dendrimer, Betulinic Acid-Chitosan and Betulinic Acid-Dendrimer for Treatment of Leishmania Major: Real-Time PCR Assay Plus. Int. J. nanomedicine 14, 7593-7607. doi:10.2147/ijn.s220410

Mehtani, D., Seth, A., Sharma, P., Maheshwari, N., Kapoor, D., Shrivastava, S. K., et al. (2019). Biomaterials for Sustained and Controlled Delivery of Small Drug Molecules, Biomaterials And Bionanotechnology. Cambridge, MA, USA: Elsevier, 89-152. doi:10.1016/b978-0-12-814427-5.00004-4

Messina, J. P., Brady, O. J., Golding, N., Kraemer, M. U., Wint, G. W., Ray, S. E., et al. (2019). The Current and Future Global Distribution and Population at Risk of Dengue. Nat. Microbiol. 4, 1508-1515. doi:10.1038/s41564-019-0476-8

Mitra, A. K., and Mawson, A. R. (2017). Neglected Tropical Diseases: Epidemiology and Global burden. Trop. Med. Infect. Dis. 2, 36. doi:10.3390/ tropicalmed 2030036

Mohapatra, S. C., Tiwari, H. K., Singla, M., Rathi, B., Sharma, A., Mahiya, K., et al. (2010). Antimalarial Evaluation of Copper (II) Nanohybrid Solids: Inhibition of Plasmepsin II, a Hemoglobin-Degrading Malarial Aspartic Protease from
Plasmodium Falciparum. JBIC J. Biol. Inorg. Chem. 15, 373-385. doi:10.1007/s00775-009-0610-9

Molyneux, D. H., and Malecela, M. N. (2011). Neglected Tropical Diseases and the Millennium Development Goals-Why the" Other Diseases" Matter: Reality versus Rhetoric, Parasites \& Vectors 4. 1-13. doi:10.1186/1756-3305-4-234

Mondal, S., Bhattacharya, P., and Ali, N. (2010). Current Diagnosis and Treatment of Visceral Leishmaniasis. Expert Rev. anti-infective Ther. 8, 919-944. doi:10.1586/eri.10.78

Morilla, M. J., Prieto, M. J., and Romero, E. L. (2005). Benznidazole vs Benznidazole in Multilamellar Liposomes: How Different They Interact with Blood Components?. Memórias do Instituto Oswaldo Cruz 100, 213-219. doi:10.1590/s0074-02762005000200017

Morilla, M. J., and Romero, E. L. (2015). Nanomedicines against Chagas Disease: an Update on Therapeutics, Prophylaxis and Diagnosis. Nanomedicine 10, 465-481. doi:10.2217/nnm.14.185

Muchiri, E. M., Thiong'o, F. W., Magnussen, P., and Ouma, J. H. (2001). A Comparative Study of Different Albendazole and Mebendazole Regimens for the Treatment of Intestinal Infections in School Children of Usigu Division, Western Kenya. J. Parasitol. 87, 413-418. doi:10.1645/0022-3395(2001)087 [0413:acsoda] 2.0.co;2

Mukherjee, S., Ray, S., and Thakur, R. (2009). Solid Lipid Nanoparticles: a Modern Formulation Approach in Drug Delivery System. Indian J. Pharm. Sci. 71, 349-358. doi:10.4103/0250-474x.57282

Mulyatno, K. C., Yamanaka, A., Yotopranoto, S., and Konishi, E. (2012). Vertical Transmission of Dengue Virus in Aedes aegypti Collected in Surabaya, Indonesia, during 2008-2011. Jpn. J. Infect. Dis. 65, 274-276. doi:10.7883/ yoken.65.274

Muraca, G., Berti, I. R., Sbaraglini, M. L., Fávaro, W. J., Durán, N., Castro, G. R., et al. (2020). Trypanosomatid-Caused Conditions: State of the Art of Therapeutics and Potential Applications of Lipid-Based Nanocarriers. Front. Chem. 8, 1077. doi:10.3389/fchem.2020.601151

Murphy, B. R., and Whitehead, S. S. (2011). Immune Response to Dengue Virus and Prospects for a Vaccine. Annu. Rev. Immunol. 29, 587-619. doi:10.1146/ annurev-immunol-031210-101315

Nagle, A. S., Khare, S., Kumar, A. B., Supek, F., Buchynskyy, A., Mathison, C. J., et al. (2014). Recent Developments in Drug Discovery for Leishmaniasis and Human African Trypanosomiasis. Chem. Rev. 114, 11305-11347. doi:10.1021/ cr500365f

Nathan, M.B., and Dayal-Drager, R. (2006). Recent Epidemiological Trends, the Global Strategy and Public Health Advances in Dengue. Rep. Dengue, $30-34$

Nayak, A. P., Tiyaboonchai, W., Patankar, S., Madhusudhan, B., and Souto, E. B. (2010). Curcuminoids-loaded Lipid Nanoparticles: Novel Approach towards Malaria Treatment. Colloids Surf. B: Biointerfaces 81, 263-273. doi:10.1016/ j.colsurfb.2010.07.020

Njoroge, M., Njuguna, N. M., Mutai, P., Ongarora, D. S., Smith, P. W., and Chibale, K. (2014). Recent Approaches to Chemical Discovery and Development against Malaria and the Neglected Tropical Diseases Human African Trypanosomiasis and Schistosomiasis. Chem. Rev. 114, 11138-11163. doi:10.1021/cr500098f

Nweze, J. A., Nweze, E. I., and Onoja, U. S. (2020). Nutrition, Malnutrition, and Leishmaniasis. Nutrition 73, 110712. doi:10.1016/j.nut.2019.110712

O'brien, J., Lee, S.-H., Gutiérrez, J. M., and Shea, K. J. (2018). Engineered Nanoparticles Bind Elapid Snake Venom Toxins and Inhibit VenomInduced Dermonecrosis. PLoS Negl. Trop. Dis. 12, e0006736. doi:10.1371/ journal.pntd.0006736

Oesterling, B. M., Gulati, A., and Joshi, M. D. (2014). Nanocarrier-Based Approaches for Treatment and Detection of Alzheimer's Disease. J. nanoscience nanotechnology 14, 137-156. doi:10.1166/jnn.2014.8906

Onyekwelu, K. C. (2019). Life Cycle of Trypanosoma Cruzi in the Invertebrate and the Vertebrate Hosts. in Biology of Trypanosoma Cruzi. (London, United Kingdom: IntechOpen).

Pacheco, P., Rodrigues, L. N. C., Ferreira, J., Gomes, A., Veríssimo, C., Louvandini, H., et al. (2018). Inclusion Complex and Nanoclusters of Cyclodextrin to Increase the Solubility and Efficacy of Albendazole. Parasitol. Res. 117, 705-712. doi:10.1007/s00436-017-5740-3

Palza, H. (2015). Antimicrobial Polymers with Metal Nanoparticles. Int. J. Mol. Sci. 16, 2099-2116. doi:10.3390/ijms16012099 
Pandian, S. R. K., Pavadai, P., Vellaisamy, S., Ravishankar, V., Palanisamy, P., Sundar, L. M., et al. (2020). Formulation and Evaluation of Rutin-Loaded Solid Lipid Nanoparticles for the Treatment of Brain Tumor. Naunyn-Schmiedeberg's Arch. Pharmacol. 394, 1-15. doi:10.1007/s00210-020-02015-9

Parvez, S., Yadagiri, G., Gedda, M. R., Singh, A., Singh, O. P., Verma, A., et al. (2020). Modified Solid Lipid Nanoparticles Encapsulated with Amphotericin B and Paromomycin: an Effective Oral Combination against Experimental Murine Visceral Leishmaniasis. Scientific Rep. 10, 1-14. doi:10.1038/s41598020-69276-5

Patel, R. P., and Joshi, J. R. (2012). An Overview on Nanoemulsion: a Novel Approach. Int. J. Pharm. Sci. Res. 3, 4640-4650. doi:10.13040/IJPSR.0975-8232

Phillips, R. O., Robert, J., Abass, K. M., Thompson, W., Sarfo, F. S., Wilson, T., et al. (2020). Rifampicin and Clarithromycin (Extended Release) versus Rifampicin and Streptomycin for Limited Buruli Ulcer Lesions: a Randomised, Open-Label, Non-inferiority Phase 3 Trial. The Lancet 395, 1259-1267. doi:10.1016/s01406736(20)30047-7

Phuphisut, O., Yoonuan, T., Sanguankiat, S., Chaisiri, K., Maipanich, W., Pubampen, S., et al. (2014). Triplex Polymerase Chain Reaction Assay for Detection of Major Soil-Transmitted Helminths, Ascaris lumbricoides, Trichuris Trichiura, Necator Americanus, in Fecal Samples. Southeast Asian J. Trop. Med. Public Health 45, 267-275.

Pinto, E. J. S., De Araujo, J. T. C., Dos Anjos Ferreira, R. M., Souto, R. N. P., Lima, L. A., De Barros Silva, P. G., et al. (2021). Larvicidal Activity, Aquatic and In Vivo Toxicity of Anacardic Acid Loaded-Zein Nanoparticles. J. Drug Deliv. Sci. Technol. 63, 102513. doi:10.1016/j.jddst.2021.102513

Ponarulselvam, S., Panneerselvam, C., Murugan, K., Aarthi, N., Kalimuthu, K., and Thangamani, S. (2012). Synthesis of Silver Nanoparticles Using Leaves of Catharanthus Roseus Linn. G. Don and Their Antiplasmodial Activities. Asian Pac. J. Trop. Biomed. 2, 574-580. doi:10.1016/s2221-1691(12)60100-2

Prayag, K., Surve, D. H., Paul, A. T., Kumar, S., and Jindal, A. B. (2020). Nanotechnological Interventions for Treatment of Trypanosomiasis in Humans and Animals. Drug Deliv. translational Res. 10, 945-961. doi:10.1007/s13346-020-00764-x

Quijia, C. R., Bonatto, C. C., Silva, L. P., Andrade, M. A., Azevedo, C. S., Lasse Silva, C., et al. (2020). Liposomes Composed by Membrane Lipid Extracts from Macrophage Cell Line as a Delivery of the Trypanocidal N, N'-Squaramide 17 towards Trypanosoma Cruzi. Materials 13, 5505. doi:10.3390/ma13235505

Radwanska, M., Vereecke, N., Deleeuw, V., Pinto, J., and Magez, S. (2018). Salivarian Trypanosomosis: A Review of Parasites Involved, Their Global Distribution and Their Interaction with the Innate and Adaptive Mammalian Host Immune System. Front. Immunol. 9, 2253. doi:10.3389/ fimmu.2018.02253

Raj, S., Sasidharan, S., Balaji, S., Dubey, V. K., and Saudagar, P. (2020). Review on Natural Products as an Alternative to Contemporary Anti-leishmanial Therapeutics. J. Proteins Proteomics 11, 135-158. doi:10.1007/s42485-020-00035-w

Rassi, A., and De Rezende, J. M. (2012). American Trypanosomiasis (Chagas Disease). Infect. Dis. Clin. 26, 275-291. doi:10.1016/j.idc.2012.03.002

Riezk, A., Van Bocxlaer, K., Yardley, V., Murdan, S., and Croft, S. L. (2020). Activity of Amphotericin B-Loaded Chitosan Nanoparticles against Experimental Cutaneous Leishmaniasis. Molecules 25, 4002. doi:10.3390/ molecules 25174002

Rigau-Pérez, J. G. (2006). Severe Dengue: The Need for New Case Definitions. Lancet Infect. Dis. 6, 297-302. doi:10.1016/S1473-3099(06)70465-0

Rock, K., Quinnell, R., Medley, G., and Courtenay, O. (2016). Progress in the Mathematical Modelling of Visceral Leishmaniasis. Adv. Parasitol. 94, 49-131. doi:10.1016/bs.apar.2016.08.001

Roellig, D. M., Ellis, A. E., and Yabsley, M. J. (2009). Oral Transmission of Trypanosoma Cruzi with Opposing Evidence for the Theory of Carnivory. J. Parasitol. 95, 360-364. doi:10.1645/ge-1740.1

Roy, P., Das, S., Bera, T., Mondol, S., and Mukherjee, A. (2010). Andrographolide Nanoparticles in Leishmaniasis: Characterization and In Vitro Evaluations. Int. J. nanomedicine 5,1113 . doi:10.2147/IJN.S14787

Saad, A. H. A., Soliman, M. I., Azzam, A. M., and Mostafa, A. B. (2015). Antiparasitic Activity of Silver and Copper Oxide Nanoparticles against Entamoeba Histolytica and Cryptosporidium Parvum Cysts. J. Egypt. Soc. Parasitol. 45, 593-602. doi:10.21608/jesp.2015.96308
Said, D., Elsamad, L., and Gohar, Y. (2012). Validity of Silver, Chitosan, and Curcumin Nanoparticles as Anti-giardia Agents. Parasitol. Res. 111, 545-554. doi:10.1007/s00436-012-2866-1

Sánchez, G., Cuellar, D., Zulantay, I., Gajardo, M., and González-Martin, G. (2002). Cytotoxicity and Trypanocidal Activity of Nifurtimox Encapsulated in Ethylcyanoacrylate Nanoparticles. Biol. Res. 35, 39-45. doi:10.4067/s071697602002000100007

Santos-Valle, A. B. C., Souza, G. R., Paes, C. Q., Miyazaki, T., Silva, A. H., Altube, M. J., et al. (2019). Nanomedicine Strategies for Addressing Major Needs in Neglected Tropical Diseases. Annu. Rev. Control. 48, 423-441. doi:10.1016/ j.arcontrol.2019.08.001

Saqib, M., Ali Bhatti, A. S., Ahmad, N. M., Ahmed, N., Shahnaz, G., Lebaz, N., et al. (2020). Amphotericin B Loaded Polymeric Nanoparticles for Treatment of Leishmania Infections. Nanomaterials 10, 1152. doi:10.3390/ nano10061152

Saravolatz, L. D., Bern, C., Adler-Moore, J., Berenguer, J., Boelaert, M., Den Boer, M., et al. (2006). Liposomal Amphotericin B for the Treatment of Visceral Leishmaniasis. Clin. Infect. Dis. 43, 917-924. doi:10.1086/507530

Savioli, L., and Albonico, M. (2004). Focus: Soil-Transmitted Helminthiasis. Nat. Rev. Microbiol. 2, 618-619. doi:10.1038/nrmicro962

Sazgarnia, A., Taheri, A. R., Soudmand, S., Parizi, A. J., Rajabi, O., and Darbandi, M. S. (2013). Antiparasitic Effects of Gold Nanoparticles with Microwave Radiation on Promastigotes and Amastigotes of Leishmania Major. Int. J. Hyperthermia 29, 79-86. doi:10.3109/02656736.2012.758875

Schmunis, G. (1991). Trypanosoma Cruzi, the Etiologic Agent of Chagas' Disease: Status in the Blood Supply in Endemic and Nonendemic Countries. Transfusion 31, 547-557. doi:10.1046/j.1537-2995.1991.31691306255.x

Schwarz, C. (1999). Solid Lipid Nanoparticles (SLNs) for Controlled Drug Delivery II. Drug Incorporation and Physicochemical Characterization. J. microencapsulation 16, 205-213. doi:10.1080/026520499289185

Seabra, A. B., and Durán, N. (2017). Nitric Oxide Donors for Treating Neglected Diseases. Nitric Oxide Donors. Elsevier, 25-53. doi:10.1016/b978-0-12-809275$0.00002-8$

Selvaraj, K., Gowthamarajan, K., Karri, V. V. S. R., Barauah, U. K., Ravisankar, V., and Jojo, G. M. (2017). Current Treatment Strategies and Nanocarrier Based Approaches for the Treatment and Management of Diabetic Retinopathy. J. Drug Target. 25, 386-405. doi:10.1080/1061186x.2017.1280809

Seremeta, K. P., Arrúa, E. C., Okulik, N. B., and Salomon, C. J. (2019). Development and Characterization of Benznidazole Nano-And Microparticles: a New Tool for Pediatric Treatment of Chagas Disease? Colloids Surf. B: Biointerfaces 177, 169-177. doi:10.1016/j.colsurfb.2019.01.039

Sherje, A. P., Jadhav, M., Dravyakar, B. R., and Kadam, D. (2018). Dendrimers: A Versatile Nanocarrier for Drug Delivery and Targeting. Int. J. pharmaceutics 548, 707-720. doi:10.1016/j.ijpharm.2018.07.030

Silva, E. F., Orsi, M., Andrade, Â. L., Domingues, R. Z., Silva, B. M., De Araújo, H. R., et al. (2012). A Tetravalent Dengue Nanoparticle Stimulates Antibody Production in Mice. J. nanobiotechnology 10, 1-5. doi:10.1186/1477-3155-10-13

Singh, L., Kruger, H. G., Maguire, G. E., Govender, T., and Parboosing, R. (2017). The Role of Nanotechnology in the Treatment of Viral Infections. Ther. Adv. Infect. Dis. 4, 105-131. doi:10.1177/2049936117713593

Sinha, J., Mukhopadhyay, S., Das, N., and Basu, M. K. (2000). Targeting of Liposomal Andrographolide to L. Donovani-Infected Macrophages In Vivo. Drug Deliv. 7, 209-213. doi:10.1080/107175400455137

Steichen, S. D., Caldorera-Moore, M., and Peppas, N. A. (2013). A Review of Current Nanoparticle and Targeting Moieties for the Delivery of Cancer Therapeutics. Eur. J. Pharm. Sci. 48, 416-427. doi:10.1016/j.ejps.2012.12.006

Stijlemans, B., De Baetselier, P., Caljon, G., Van Den Abbeele, J., Van Ginderachter, J. A., and Magez, S. (2017). Nanobodies as Tools to Understand, Diagnose, and Treat African Trypanosomiasis. Front. Immunol. 8, 724. doi:10.3389/ fimmu.2017.00724

$\mathrm{Su}, \mathrm{S}$., and Kang, P. M. (2020). Systemic Review of Biodegradable Nanomaterials in Nanomedicine. Nanomaterials 10, 656. doi:10.3390/nano10040656

Subhaswaraj, P., Syed, A., and Siddhardha, B. (2020). Novel Nanotherapeutics as Next-Generation Anti-infective Agents: Current Trends and Future Prospectives. Curr. Drug Discov. Tech. 17, 457-468. doi:10.2174/ 1570163816666190715120708 
Subramaniam, J., Murugan, K., Panneerselvam, C., Kovendan, K., Madhiyazhagan, P., Dinesh, D., et al. (2016). Multipurpose Effectiveness of Couroupita Guianensis-Synthesized Gold Nanoparticles: High Antiplasmodial Potential, Field Efficacy against Malaria Vectors and Synergy with Aplocheilus Lineatus Predators. Environ. Sci. Pollut. Res. 23, 7543-7558. doi:10.1007/s11356-0156007-0

Sun, N., and Amon, J. J. (2018). Addressing Inequity: Neglected Tropical Diseases and Human Rights. Health Hum. Rights 20, 11-25.

Torabi, N., Dobakhti, F., and Haniloo, A. (2018). Albendazole and Praziquantel Chitosan Nanoparticles: Preparation, Characterization, and In Vitro Release Study. Iranian J. Sci. Technol. Trans. A: Sci. 42, 1269-1275. doi:10.1007/s40995017-0150-z

Torres-Guerrero, E., Quintanilla-Cedillo, M. R., Ruiz-Esmenjaud, J., and Arenas, R. (2017). Leishmaniasis: a Review, F1000Res. 6, 750. doi:10.12688/ f1000research.11120.1

Tripathy, S., Das, S., Chakraborty, S. P., Sahu, S. K., Pramanik, P., and Roy, S. (2012). Synthesis, Characterization of Chitosan-Tripolyphosphate Conjugated Chloroquine Nanoparticle and its In Vivo Anti-malarial Efficacy against Rodent Parasite: A Dose and Duration Dependent Approach. Int. J. pharmaceutics 434, 292-305. doi:10.1016/ j.ijpharm.2012.05.064

Uchegbu, I. F., and Vyas, S. P. (1998). Non-ionic Surfactant Based Vesicles (Niosomes) in Drug Delivery. Int. J. pharmaceutics 172, 33-70. doi:10.1016/ s0378-5173(98)00169-0

Unciti-Broceta, J. D., Arias, J. L., Maceira, J., Soriano, M., Ortiz-González, M., Hernández-Quero, J., et al. (2015). Specific Cell Targeting Therapy Bypasses Drug Resistance Mechanisms in African Trypanosomiasis. Plos Pathog. 11, e1004942. doi:10.1371/journal.ppat.1004942

Varela-Aramburu, S., Ghosh, C., Goerdeler, F., Priegue, P., Moscovitz, O., and Seeberger, P. H. (2020). Targeting and Inhibiting Plasmodium Falciparum Using Ultra-small Gold Nanoparticles. ACS Appl. Mater. Inter. 12, 43380-43387. doi:10.1021/acsami.0c09075

Vashist, A., Kaushik, A., Vashist, A., Jayant, R. D., Tomitaka, A., Ahmad, S., et al. (2016). Recent Trends on Hydrogel Based Drug Delivery Systems for Infectious Diseases. Biomater. Sci. 4, 1535-1553. doi:10.1039/c6bm00276e

Venier-Julienne, M., Vouldoukis, I., Monjour, L., and Benoit, J. (1995). In Vitro study of the Anti-leishmanial Activity of Biodegradable Nanoparticles. J. Drug Target. 3, 23-29. doi:10.3109/10611869509015929

Vermelho, A. B., Capaci, G. R., Rodrigues, I. A., Cardoso, V. S., Mazotto, A. M., and Supuran, C. T. (2017). Carbonic Anhydrases from Trypanosoma and Leishmania as Anti-protozoan Drug Targets. Bioorg. Med. Chem. 25, 1543-1555. doi:10.1016/j.bmc.2017.01.034

Volpedo, G., Costa, L., Ryan, N., Halsey, G., Satoskar, A., and Oghumu, S. (2019). Nanoparticulate Drug Delivery Systems for the Treatment of Neglected Tropical Protozoan Diseases. J. Venomous Anim. Toxins including Trop. Dis. 25, 1-14. doi:10.1590/1678-9199-JVATITD-1441-18
Weaver, S. C., and Vasilakis, N. (2009). Molecular Evolution of Dengue Viruses Contributions of Phylogenetics to Understanding the History and Epidemiology of the Preeminent Arboviral Disease. Infect. Genet. Evol. 9, 523-540. doi:10.1016/j.meegid.2009.02.003

Weng, H.-B., Chen, H.-X., and Wang, M.-W. (2018). Innovation in Neglected Tropical Disease Drug Discovery and Development. Infect. Dis. poverty 7, 1-9. doi:10.1186/s40249-018-0444-1

Wijerathna, T., Gunathilaka, N., Gunawardana, K., and Rodrigo, W. (2017). Potential Challenges of Controlling Leishmaniasis in Sri Lanka at a Disease Outbreak. Biomed. Res. Int. 2017, 1-10. doi:10.1155/2017/6931497

World Health Organization (2015). Investing to Overcome the Global Impact of Neglected Tropical Diseases: Third WHO Report on Neglected Tropical Diseases 2015. Geneva, Switzerland: World Health Organization.

World Health Organization (2018). Status of Endemicity of Visceral Leishmaniasis, Worldwide. 2015. Geneva, Switzerland: World Health Organization.

World Health Organization (2013). Sustaining the Drive to Overcome the Global Impact of Neglected Tropical Diseases: Second WHO Report on Neglected Diseases. Geneva, Switzerland: World Health Organization.

World Health Organization (2010). Working to Overcome the Global Impact of Neglected Tropical Diseases: First WHO Report on Neglected Tropical Diseases. Geneva, Switzerland: World Health Organization.

Yasinzai, M., Khan, M., Nadhman, A., and Shahnaz, G. (2013). Drug Resistance in Leishmaniasis: Current Drug-Delivery Systems and Future Perspectives. Future Med. Chem. 5, 1877-1888. doi:10.4155/fmc.13.143

Zhang, L., Gu, F., Chan, J., Wang, A., Langer, R., and Farokhzad, O. (2008). Nanoparticles in Medicine: Therapeutic Applications and Developments. Clin. Pharmacol. Ther. 83, 761-769. doi:10.1038/sj.clpt.6100400

Zhang, Y., Tu, J., Wang, D., Zhu, H., Maity, S. K., Qu, X., et al. (2018). Programmable and Multifunctional DNA-based Materials for Biomedical Applications. Adv. Mater. 30, 1703658. doi:10.1002/adma.201703658

Zinselmeyer, B. H., Mackay, S. P., Schatzlein, A. G., and Uchegbu, I. F. (2002). The Lower-Generation Polypropylenimine Dendrimers Are Effective GeneTransfer Agents. Pharm. Res. 19, 960-967. doi:10.1023/a:1016458104359

Conflict of Interest: The authors declare that the research was conducted in the absence of any commercial or financial relationships that could be construed as a potential conflict of interest.

Copyright (C) 2021 Pandian, Panneerselvam, Pavadai, Govindaraj, Ravishankar, Palanisamy, Sampath, Sankaranarayanan and Kunjiappan. This is an open-access article distributed under the terms of the Creative Commons Attribution License (CC $B Y)$. The use, distribution or reproduction in other forums is permitted, provided the original author(s) and the copyright owner(s) are credited and that the original publication in this journal is cited, in accordance with accepted academic practice. No use, distribution or reproduction is permitted which does not comply with these terms. 